\title{
Quantum and thermal ionic motion, oxygen isotope effect, and superexchange distribution in La2CuO4
}

Haefliger, P. S.; Gerber, S.; Pramod, R.; Schnells, V. I.; dalla Piazza, B.; Chati, R.; Pomjakushin, V.; Conder, K.; Pomjakushina, E.; Le Dreau, L.

Total number of authors:

14

Published in:

Physical Review B Condensed Matter

Link to article, DOI:

10.1103/PhysRevB.89.085113

Publication date:

2014

Document Version

Publisher's PDF, also known as Version of record

Link back to DTU Orbit

Citation (APA):

Haefliger, P. S., Gerber, S., Pramod, R., Schnells, V. I., dalla Piazza, B., Chati, R., Pomjakushin, V., Conder, K., Pomjakushina, E., Le Dreau, L., Christensen, N. B., Syljuasen, O. F., Normand, B., \& Rønnow, H. M. (2014).

Quantum and thermal ionic motion, oxygen isotope effect, and superexchange distribution in $\mathrm{La}_{2} \mathrm{CuO}_{4}$. Physical Review B Condensed Matter, 89(8), [085113]. https://doi.org/10.1103/PhysRevB.89.085113

\section{General rights}

Copyright and moral rights for the publications made accessible in the public portal are retained by the authors and/or other copyright owners and it is a condition of accessing publications that users recognise and abide by the legal requirements associated with these rights.

- Users may download and print one copy of any publication from the public portal for the purpose of private study or research.

- You may not further distribute the material or use it for any profit-making activity or commercial gain

- You may freely distribute the URL identifying the publication in the public portal 


\title{
Quantum and thermal ionic motion, oxygen isotope effect, and superexchange distribution in $\mathrm{La}_{2} \mathrm{CuO}_{4}$
}

\author{
P. S. Häfliger, ${ }^{1}$ S. Gerber,,${ }^{1,2,}$ R. Pramod,${ }^{3}$ V. I. Schnells, ${ }^{3}$ B. dalla Piazza, ${ }^{3}$ R. Chati,,${ }^{1}$ V. Pomjakushin, ${ }^{2}$ K. Conder ${ }^{4}$ \\ E. Pomjakushina, ${ }^{4}$ L. Le Dreau, ${ }^{4,5}$ N. B. Christensen, ${ }^{2,6}$ O. F. Syljuåsen, ${ }^{7}$ B. Normand, ${ }^{8}$ and H. M. Rønnow ${ }^{3}$ \\ ${ }^{1}$ Laboratory for Solid State Physics, ETH Zurich, CH-8093 Zurich, Switzerland \\ ${ }^{2}$ Laboratory for Neutron Scattering, Paul Scherrer Institute, CH-5232 Villigen PSI, Switzerland \\ ${ }^{3}$ Laboratory for Quantum Magnetism, École Polytechnique Fédérale de Lausanne (EPFL), 1015 Lausanne, Switzerland \\ ${ }^{4}$ Laboratory for Developments and Methods, Paul Scherrer Institute, CH-5232 Villigen PSI, Switzerland \\ ${ }^{5}$ Laboratory of Soft Chemistry and Reactivity, University of Rennes 1 UMR 6226, 35042 Rennes, France \\ ${ }^{6}$ Department of Physics, Technical University of Denmark (DTU), DK-2800 Kongens Lyngby, Denmark \\ ${ }^{7}$ Department of Physics, University of Oslo, P. O. Box 1048 Blindern, N-0316 Oslo, Norway \\ ${ }^{8}$ Department of Physics, Renmin University of China, Beijing 100872, People's Republic of China \\ (Received 2 December 2013; revised manuscript received 22 January 2014; published 18 February 2014)
}

\begin{abstract}
We study the zero-point and thermal ionic motion in $\mathrm{La}_{2} \mathrm{CuO}_{4}$ by means of high-resolution neutron-diffraction experiments. Our results demonstrate anisotropic motion of $\mathrm{O}$ and, to a lesser extent, $\mathrm{Cu}$ ions, both consistent with the structure of coupled $\mathrm{CuO}_{6}$ octahedra, and quantify the relative effects of zero-point and thermal contributions to ionic motion. By substitution of ${ }^{18} \mathrm{O}$, we find that the oxygen isotope effect on the lattice dimensions is small and negative $(-0.01 \%)$, while the isotope effect on the ionic displacement parameters is significant ( -6 to $50 \%$ ). We use our results as input for theoretical estimates of the distribution of magnetic interaction parameters, $J$, in an effective one-band model for the cuprate plane. We find that ionic motion causes only small (1\%) effects on the average value $\langle J\rangle$, which vary with temperature and $\mathrm{O}$ isotope, but results in dramatic (10-20\%) fluctuations in $J$ values that are subject to significant $(8-12 \%)$ isotope effects. We demonstrate that this motional broadening of $J$ can have substantial effects on certain electronic and magnetic properties in cuprates.
\end{abstract}

DOI: 10.1103/PhysRevB.89.085113

PACS number(s): 61.05.fm, 74.25.-q, 74.72.Cj, 75.30.Et

\section{INTRODUCTION}

High-temperature superconductivity remains one of the fundamental challenges in condensed-matter physics. More than 25 years of intensive experimental and theoretical studies [1] have brought new and profound understanding to many branches of the physics of strongly correlated electrons. However, many mysteries remain concerning some basic issues such as the pairing mechanism, the role of the lattice, and the importance of structural and electronic homogeneity. Here we address perhaps the most basic unanswered question of all, namely, where are the atoms? It is known that the atoms are quantum mechanical entities, subject to a range of quantum fluctuations, including positional ones. Knowing the positions of the atoms, on time scales relevant to electronic processes, is essential to the understanding of any kind of model for the complex cuprate phase diagram or the mechanism for superconducting pairing.

Quantum fluctuations play an essential role in the electronic and magnetic properties of the cuprates [2]. In the parent antiferromagnetic phase, the suppressed moment [3] and the zone-boundary spin-wave dispersion [4] are the fingerprints of intrinsically quantum mechanical effects typical of a twodimensional (2D) $S=1 / 2$ Heisenberg antiferromagnet [5]. The motion of electrons in this quantum spin medium, including their pairing tendencies, is then very strongly renormalized by fluctuation effects [6]. The importance of

\footnotetext{
*Present address: Stanford Institute for Materials and Energy Sciences, SLAC National Accelerator Laboratory and Stanford University, Menlo Park, California 94025, USA.
}

quantum fluctuations is manifest not only in spin space but also in real space, in the form of zero-point motion. In $\mathrm{C}_{60}$ materials, the effects of zero-point motion are found to be very significant [7], in the sense that they may be responsible for a substantial renormalization of the electron-phonon coupling and hence of superconductivity. In the cuprate materials, where superconducting transition temperatures $\left(T_{c}\right)$ are very high, neither the effect of zero-point motion nor of thermal ionic motion has yet been addressed experimentally.

The isotope effect on $T_{c}[8,9]$ is possibly the clearest signature of the role of the lattice and is one of the keys to conventional superconductivity. A purely phononic pairing mechanism will give a very characteristic dependence of superconducting properties on the mass of the participating ions [10]. In cuprates, where the contributions to the pairing mechanism have not been quantified, isotope effects on the electronic properties are well known but complex: while the pseudogap temperature rises [11] with ${ }^{18} \mathrm{O}$ isotope substitution, the superconducting transition temperature decreases [12]. In contrast, only little is known regarding isotope effects on magnetic properties. Combined muon spin-rotation and magnetization studies [13] found that the antiferromagnetic and spin-glass ordering temperatures in $\mathrm{Y}_{1-x} \mathrm{Pr}_{x} \mathrm{Ba}_{2} \mathrm{Cu}_{3} \mathrm{O}_{7-\delta}$ exhibit a large oxygen isotope effect (OIE) in the regime where superconductivity and antiferromagnetic order coexist. A very large OIE on the spin-glass temperature has also been found in $\mathrm{Mn}$-doped $\mathrm{La}_{1-x} \mathrm{Sr}_{x} \mathrm{CuO}_{4}$ at low doping [14]. These unusual effects could arise from the isotope-dependent mobility of the charge carriers [15]. For undoped $\mathrm{La}_{2} \mathrm{CuO}_{4}$, the Néel temperature $T_{N}$ was reported to decrease slightly upon oxygen isotope substitution [16], a 
result assumed to originate from structural changes [17]. To date, however, the OIE has been measured only for the lattice constants and the consequent orthorhombicity, and there is in particular no information concerning either zero-point or thermal ionic motion.

The electronic and magnetic properties of an interacting system depend fundamentally on superexchange processes between electronic orbitals, which mediate the electron hopping and spin-fluctuation energy scales, and therefore on the dimensions and geometry of the host lattice. Thus, both zeropoint (quantum) and thermal ionic motion can have significant consequences for the physical properties of a system, including its superconductivity. Quite generally, the time scale for ionic motion in condensed-matter systems is much longer than that for electronic processes. However, this situation (the Born approximation) may break down for "low-energy" electronic properties, especially superconductivity, in systems with high phonon energy scales. These observations suggest the importance of a careful and comparative study of ionic motion in materials such as cuprates.

To investigate these open questions, we have performed a high-resolution neutron-diffraction study of high-quality $\mathrm{La}_{2} \mathrm{CuO}_{4}$ powders. Because the neutron cross section is directly proportional to the Debye-Waller factor, neutron diffraction is an excellent probe of zero-point and thermal motion. We obtain data suitable for a detailed analysis of both structural and thermal properties and, by using powder of high isotopic substitution, of the OIE on the measured quantities. Our results provide essential input for modeling the effects of the quantum and thermal fluctuations in ionic positions on the electronic and magnetic properties of the cuprate plane. In Sec. II, we present the details of our samples, experiments, and structural refinement results. Section III discusses the isotope effect and Sec. IV analyzes the effective electronic models required to incorporate our measured motional effects into the physics of cuprates. A summary is provided in Sec. V.

\section{EXPERIMENT}

\section{A. Sample preparation and characterization}

Polycrystalline samples of $\mathrm{La}_{2} \mathrm{CuO}_{4}$ were prepared using conventional solid-state synthesis. Oxygen isotope exchange was performed by annealing of the sample in ${ }^{18} \mathrm{O}_{2}$ gas (Eurisotop, $97 \%$ isotope enrichment) at $850 \mathrm{C}$ for 30 hours [18]. The isotope content was determined by in situ mass spectroscopy measurements of the isotope composition of the $\mathrm{O}_{2}$ gas in equilibrium with the sample. After the exchange process, the isotope enrichment was evaluated by thermal analysis, where the change in mass of the sample was measured during an oxygen isotope reverse exchange performed in ordinary oxygen (replacing ${ }^{18} \mathrm{O}$ by ${ }^{16} \mathrm{O}$ ). The isotope enrichment was found to be $78 \pm 2 \%$.

The oxygen stoichiometry coefficient in both ${ }^{16} \mathrm{O}$ and ${ }^{18} \mathrm{O}$ samples was determined by thermogravimetric hydrogen reduction $[18,19]$. Both samples were found to have an oxygen content of $4.004 \pm 0.005$, and hence to be oxygen stoichiometric within the experimental error of the determination procedure.

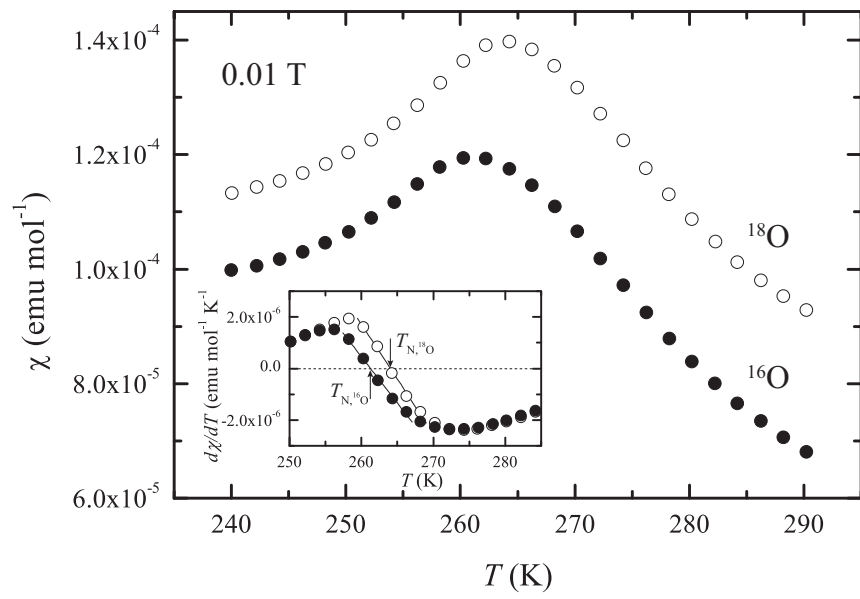

FIG. 1. Susceptibility as a function of temperature for $\mathrm{La}_{2} \mathrm{Cu}^{16} \mathrm{O}_{4}$ (solid circles) and $\mathrm{La}_{2} \mathrm{Cu}^{18} \mathrm{O}_{4}$ (open circles). The inset shows the derivative of the susceptibility used to deduce the value of $T_{N}$ for both cases.

\section{B. Magnetization measurements}

Magnetization measurements were performed using a Quantum Design MPMS in fields ranging from $20 \mathrm{mT}$ to $6 \mathrm{~T}$ at temperatures between 4 and $300 \mathrm{~K}$ on $\mathrm{La}_{2} \mathrm{Cu}^{16} \mathrm{O}_{4}$ and $\mathrm{La}_{2} \mathrm{Cu}^{18} \mathrm{O}_{4}$. The magnetic susceptibility $\chi(T)$ obtained in an applied field of $0.1 \mathrm{~T}$ is shown in Fig. 1 for both ${ }^{16} \mathrm{O}$ and ${ }^{18} \mathrm{O}$ samples. Clear peaks are observed at the onset of antiferromagnetic order in both cases. Several factors contribute to the rounding of these peaks, among which the powder nature of the sample is the most important. The derivatives $d \chi / d T$, shown in the inset of Fig. 1, give definitive peak values, which we take as the Néel temperatures of the two samples, $T_{N}=261.4 \pm 0.1 \mathrm{~K}$ for $\mathrm{La}_{2} \mathrm{Cu}^{16} \mathrm{O}_{4}$ and $T_{N}=263.8 \pm 0.1 \mathrm{~K}$ for $\mathrm{La}_{2} \mathrm{Cu}^{18} \mathrm{O}_{4}$.

\section{Neutron diffraction}

Neutron diffraction was performed on the high-resolution powder diffractometer HRPT [20] at the Swiss spallation neutron source SINQ [21], located at the Paul Scherrer Institute in Switzerland. The experiments were carried out at a wavelength $\lambda=1.1545 \AA$. The $\mathrm{La}_{2} \mathrm{Cu}^{16} \mathrm{O}_{4}$ and $\mathrm{La}_{2} \mathrm{Cu}^{18} \mathrm{O}_{4}$ samples were each placed in a 8 -mm-diameter vanadium container, which was mounted into a closed-cycle refrigerator reaching temperatures between 15 and $290 \mathrm{~K}$. High-statistics data were taken at 15 and $290 \mathrm{~K}\left(3.5 \times 10^{7}\right.$ counts $)$, whereas points at temperatures $15<T<290 \mathrm{~K}$ were obtained with intermediate statistics $\left(2.5 \times 10^{6}\right.$ counts $)$.

The diffraction study showed that the samples of $\mathrm{La}_{2} \mathrm{Cu}^{16} \mathrm{O}_{4}$ and $\mathrm{La}_{2} \mathrm{Cu}^{18} \mathrm{O}_{4}$ both crystallized in the orthorhombic space group $\mathrm{Bmba}$ (No. 64), with atomic positions $\mathrm{O} 1=(1 / 4,1 / 4, z), \mathrm{O} 2=(0, y, z), \mathrm{La}=(0, y, z)$, and $\mathrm{Cu}=$ $(0,0,0)$. These results are fully consistent with earlier reports [22].

The intensity patterns obtained from neutron diffraction were refined using the program FULLPROF [23], and the errors shown in Tables I-III and Figs. 2 and 3 are those provided by the FULLPROF refinement. The diffraction patterns and the corresponding structural refinement of the $15 \mathrm{~K}$ 
TABLE I. Lattice parameters, $\mathrm{Cu}-\mathrm{O} 1$ bond lengths, $\mathrm{Cu}-\mathrm{O} 1-\mathrm{Cu}$ bond angles, and atomic coordinates for $\mathrm{La}_{2} \mathrm{Cu}^{16} \mathrm{O}_{4}$ and $\mathrm{La}_{2} \mathrm{Cu}^{18} \mathrm{O}_{4}$ at temperatures of 15 and $290 \mathrm{~K}$, obtained from an isotropic refinement. In the space group Bmba (isomorphic to Cmca, No. 64) used here, the atomic positions are $(0, y, z)$ for $\mathrm{La},(0,0,0)$ for $\mathrm{Cu},(1 / 4,1 / 4, z)$ for $\mathrm{O} 1$, and $(0, y, z)$ for $\mathrm{O} 2$.

\begin{tabular}{|c|c|c|c|c|c|}
\hline & $a(\AA)$ & $b(\AA)$ & $c(\AA)$ & $d_{\mathrm{Cu}-\mathrm{O} 1}(\AA)$ & $\theta_{\mathrm{Cu}-\mathrm{O} 1-\mathrm{Cu}}(\mathrm{deg})$ \\
\hline \multicolumn{6}{|l|}{$15 \mathrm{~K}$} \\
\hline${ }^{16} \mathrm{O}$ & $5.33305(2)$ & $5.41783(3)$ & $13.10260(7)$ & $1.90369(5)$ & $173.434(2)$ \\
\hline${ }^{18} \mathrm{O}$ & $5.33185(3)$ & $5.41751(3)$ & $13.09979(8)$ & $1.90343(5)$ & $173.419(2)$ \\
\hline \multicolumn{6}{|l|}{$290 \mathrm{~K}$} \\
\hline${ }^{16} \mathrm{O}$ & $5.35479(3)$ & $5.40338(3)$ & $13.14810(9)$ & $1.90434(5)$ & $174.095(2)$ \\
\hline \multirow[t]{2}{*}{${ }^{18} \mathrm{O}$} & $5.35385(3)$ & $5.40283(3)$ & $13.14614(9)$ & $1.90401(5)$ & $174.174(2)$ \\
\hline & $y(\mathrm{La})$ & $z(\mathrm{La})$ & $z(\mathrm{O} 1)$ & $y(\mathrm{O} 2)$ & $z(\mathrm{O} 2)$ \\
\hline \multicolumn{6}{|l|}{$15 \mathrm{~K}$} \\
\hline${ }^{16} \mathrm{O}$ & $-0.00839(13)$ & $0.36149(4)$ & $-0.00832(6)$ & $0.04101(12)$ & $0.18302(8)$ \\
\hline${ }^{18} \mathrm{O}$ & $-0.00855(14)$ & $0.36158(4)$ & $-0.00834(6)$ & $0.04110(13)$ & $0.18293(8)$ \\
\hline \multicolumn{6}{|l|}{$290 \mathrm{~K}$} \\
\hline${ }^{16} \mathrm{O}$ & $-0.00683(19)$ & $0.36135(4)$ & $-0.00746(8)$ & $0.03479(17)$ & $0.18308(9)$ \\
\hline${ }^{18} \mathrm{O}$ & $-0.00655(20)$ & $0.36138(4)$ & $-0.00736(8)$ & $0.03463(17)$ & $0.18291(9)$ \\
\hline
\end{tabular}

TABLE II. Motional parameters $U_{i j}$ for $\mathrm{La}_{2} \mathrm{Cu}^{16} \mathrm{O}_{4}$ and $\mathrm{La}_{2} \mathrm{Cu}^{18} \mathrm{O}_{4}$, expressed in units of $10^{-3} \AA^{2}$, as obtained from a fully isotropic structural refinement (left), a refinement where only $\mathrm{O} 1$ and $\mathrm{O} 2$ were refined anisotropically (middle), and a fully anisotropic refinement (right). Data for 290 and $15 \mathrm{~K}$ are shown, respectively, in the top and bottom halves of the table. No convergent fit was obtained for a fully anisotropic fit at $15 \mathrm{~K}$ (see text). The shape of the symmetric $U_{i j}$ tensor $(i, j=1,2,3)$ is given by the symmetry of the space group $B m b a$, whence some components are always zero; the isotropic motional parameter $U_{\text {iso }}=\sum_{i} U_{i i} / 3$.

\begin{tabular}{|c|c|c|c|c|c|c|c|c|c|c|c|c|}
\hline \multirow[b]{2}{*}{$290 \mathrm{~K}$} & \multirow{2}{*}{$\begin{array}{c}\text { Isotropic } \\
U_{\text {iso }}\end{array}$} & \multicolumn{6}{|c|}{ Half anisotropic } & \multicolumn{5}{|c|}{ Fully anisotropic } \\
\hline & & $U_{\text {iso }}$ & $U_{11}$ & $U_{22}$ & $U_{33}$ & $U_{12}$ & $U_{23}$ & $U_{11}$ & $U_{22}$ & $U_{33}$ & $U_{12}$ & $U_{23}$ \\
\hline${ }^{16} \mathrm{O} \mathrm{La}$ & $4.6(1)$ & $4.8(1)$ & & & & & & $5.2(2)$ & $5.6(2)$ & $3.6(2)$ & & $-0.2(3)$ \\
\hline${ }^{18} \mathrm{O}$ & $5.2(1)$ & $5.3(1)$ & & & & & & $5.4(2)$ & $6.2(2)$ & $4.5(2)$ & & $-0.4(3)$ \\
\hline${ }^{16} \mathrm{O} \mathrm{Cu}$ & $4.1(1)$ & $4.3(1)$ & & & & & & $1.6(3)$ & $3.8(3)$ & $7.6(3)$ & & $0.7(4)$ \\
\hline${ }^{18} \mathrm{O}$ & $4.6(1)$ & $4.7(1)$ & & & & & & $2.1(3)$ & $4.4(3)$ & $8.0(4)$ & & $0.0(5)$ \\
\hline${ }^{16} \mathrm{O} \mathrm{O} 1$ & $6.6(1)$ & & $4.2(2)$ & $4.9(3)$ & $11.9(4)$ & $-1.6(2)$ & & $4.0(3)$ & $4.7(3)$ & $11.8(4)$ & $-1.5(2)$ & \\
\hline${ }^{18} \mathrm{O}$ & $6.0(3)$ & & $3.9(3)$ & $4.5(3)$ & $10.7(4)$ & $-1.7(2)$ & & $3.8(3)$ & $4.2(3)$ & $10.7(4)$ & $-1.6(2)$ & \\
\hline${ }^{16} \mathrm{O} \mathrm{O} 2$ & $12.3(2)$ & & $18.6(3)$ & 13.1(4) & $6.0(3)$ & & $0.9(4)$ & $18.9(4)$ & 13.1(5) & $5.1(3)$ & & $0.4(4)$ \\
\hline${ }^{18} \mathrm{O}$ & $11.6(2)$ & & $17.7(3)$ & 11.7(4) & $5.9(3)$ & & $1.0(4)$ & $18.4(4)$ & $11.4(5)$ & $4.8(3)$ & & $0.3(4)$ \\
\hline $15 \mathrm{~K}$ & $U_{\text {iso }}$ & $U_{\text {iso }}$ & $U_{11}$ & $U_{22}$ & $U_{33}$ & $U_{12}$ & $U_{23}$ & $U_{11}$ & $U_{22}$ & $U_{33}$ & $U_{12}$ & $U_{23}$ \\
\hline${ }^{16} \mathrm{O} \mathrm{La}$ & $1.0(1)$ & $1.0(1)$ & & & & & & & & & & \\
\hline${ }^{18} \mathrm{O}$ & $1.5(1)$ & $1.5(1)$ & & & & & & & & & & \\
\hline${ }^{16} \mathrm{O} \mathrm{Cu}$ & $1.4(1)$ & $1.4(1)$ & & & & & & & & & & \\
\hline${ }^{18} \mathrm{O}$ & $1.8(1)$ & $1.9(1)$ & & & & & & & & & & \\
\hline${ }^{16} \mathrm{O} \mathrm{O} 1$ & $3.0(1)$ & & $2.0(3)$ & $3.6(3)$ & $2.9(3)$ & $-0.4(2)$ & & & & & & \\
\hline${ }^{18} \mathrm{O}$ & $2.3(1)$ & & $1.4(3)$ & $2.9(3)$ & $2.3(3)$ & $-0.3(2)$ & & & & & & \\
\hline${ }^{16} \mathrm{O} \mathrm{O} 2$ & $5.3(1)$ & & $6.2(3)$ & $5.4(3)$ & $3.9(3)$ & & $-0.9(3)$ & & & & & \\
\hline${ }^{18} \mathrm{O}$ & $4.3(1)$ & & $5.3(3)$ & $4.2(3)$ & $3.0(3)$ & & $-0.6(3)$ & & & & & \\
\hline
\end{tabular}

TABLE III. OIE on the lattice parameters, on the $\mathrm{Cu}-\mathrm{O} 1-\mathrm{Cu}$ bond distance and angle, and on the isotropic ionic motion parameters in $\mathrm{La}_{2} \mathrm{CuO}_{4}$.

\begin{tabular}{|c|c|c|c|c|c|c|c|c|c|}
\hline \multirow[b]{2}{*}{$T$} & \multicolumn{3}{|c|}{ Lattice parameters } & \multicolumn{2}{|c|}{ Bond parameters } & \multicolumn{4}{|c|}{$\Delta B_{\text {iso }}$} \\
\hline & $\Delta a\left[10^{-4}\right]$ & $\Delta b\left[10^{-4}\right]$ & $\Delta c\left[10^{-4}\right]$ & $\Delta d_{\mathrm{Cu}-\mathrm{O} 1}\left[10^{-4}\right]$ & $\Delta\left(180-\theta_{\mathrm{Cu}-\mathrm{O} 1-\mathrm{Cu}}\right)$ & $\mathrm{La}$ & $\mathrm{Cu}$ & O1 & $\mathrm{O} 2$ \\
\hline $15 \mathrm{~K}$ & $-2.25(7)$ & $-0.59(8)$ & $-2.14(8)$ & $-1.37(37)$ & $0.0023(4)$ & $0.50(18)$ & $0.29(12)$ & $-0.23(4)$ & $-0.19(2)$ \\
\hline $290 \mathrm{~K}$ & $-1.76(8)$ & $-1.02(8)$ & $-1.49(10)$ & $-1.73(37)$ & $-0.0134(5)$ & $0.13(3)$ & $0.12(4)$ & $-0.09(2)$ & $-0.06(2)$ \\
\hline
\end{tabular}




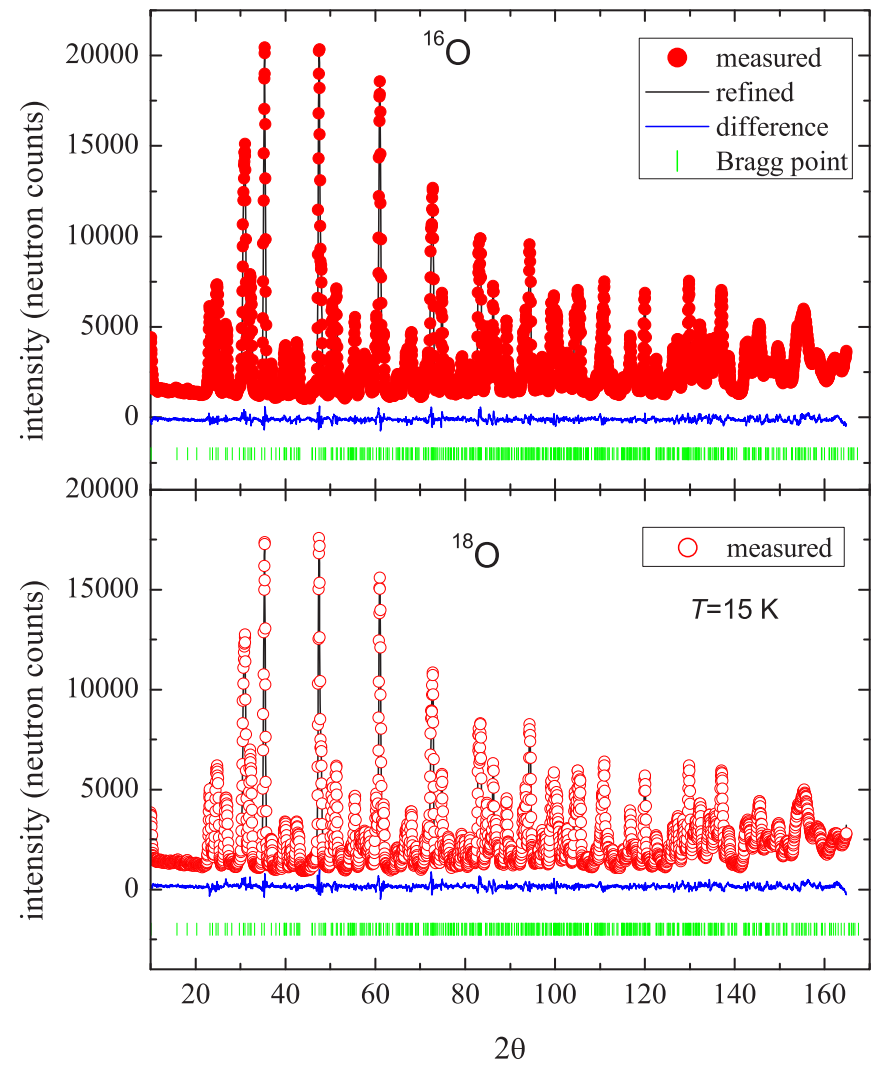

FIG. 2. (Color online) Neutron-diffraction data at $15 \mathrm{~K}$ for $\mathrm{La}_{2} \mathrm{Cu}^{16} \mathrm{O}_{4}$ (upper panel) and $\mathrm{La}_{2} \mathrm{Cu}^{18} \mathrm{O}_{4}$ (lower panel). The solid black line corresponds to a Rietvield refinement, and the difference between measured and calculated profiles is shown on the same scale. Tick marks below each panel represent the positions of allowed Bragg reflections in the Bmba space group.

data are depicted in Fig. 2. We emphasize that the samples were single phased. The oxygen stoichiometry obtained from the structural refinement confirmed the results obtained by hydrogen reduction. The small excess oxygen concentration is consistent with the fact that rounding of the susceptibility peak shown in Fig. 1 is rather weak [2].

The ionic displacement parameters enter the refinement through the Debye-Waller factor, whose determination requires high data quality and good resolution, particularly at large scattering angles. At $290 \mathrm{~K}$, reliable refinements were achieved, which allowed us to determine a fully anisotropic set of parameters for the ionic motion. However, because the ionic motion is smaller at $15 \mathrm{~K}$, its effect on the diffraction pattern was insufficiently strong for a reliable refinement of all 16 ionic motion parameters. Reliable refinements could be obtained only by constraining the motion of the $\mathrm{Cu}$ and $\mathrm{La}$ ions to be isotropic, while still allowing anisotropic motion of the $\mathrm{O}$ ions. To enable a meaningful comparison between the 15 and $290 \mathrm{~K}$ data, we performed the same "half-anisotropic" refinement at $290 \mathrm{~K}$, finding the parameters extracted for the $\mathrm{O}$ ions to be very close to those given by the fully anisotropic refinement, and thus supporting the consistency of our results. Finally, to compare with previous results for the static lattice parameters and to visualize the temperature dependence of the motional parameters, a set of refinements was also performed

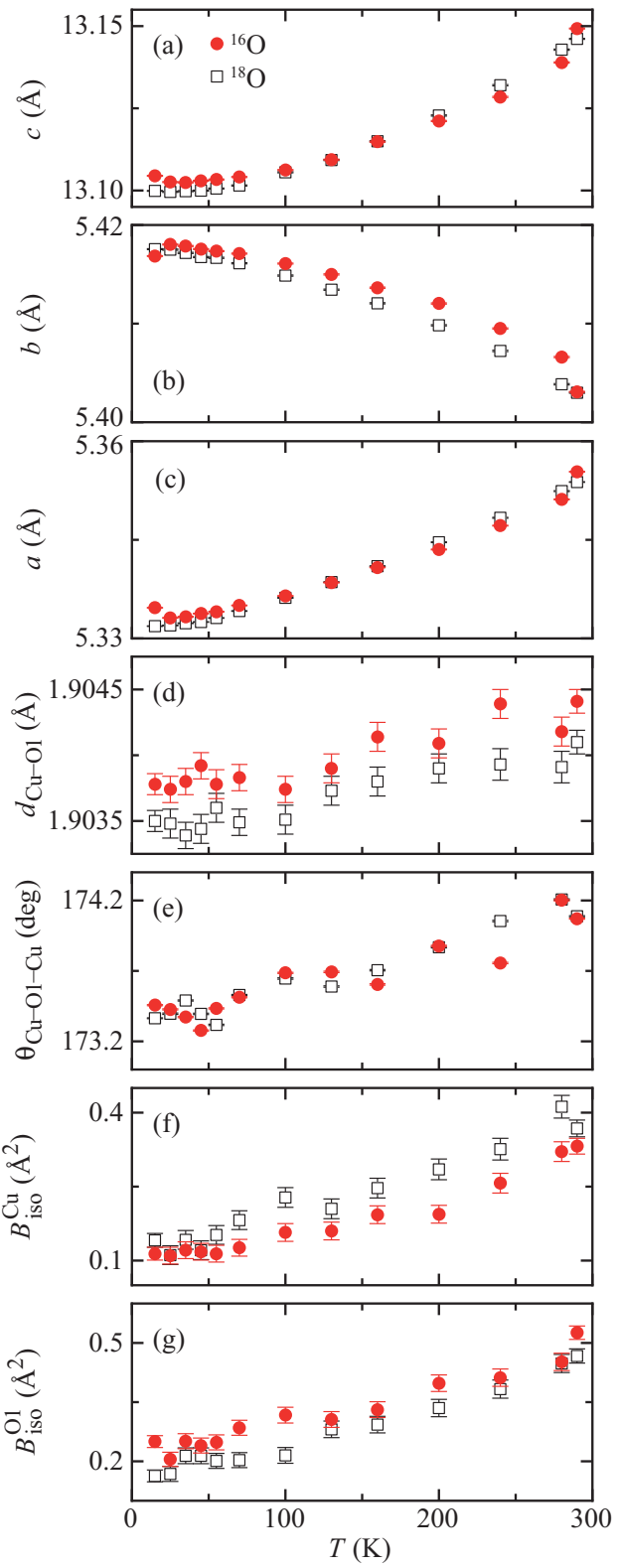

FIG. 3. (Color online) Temperature dependence of structural parameters extracted from refinements using isotropic ionic motion parameters for $\mathrm{La}_{2} \mathrm{Cu}^{16} \mathrm{O}_{4}$ (solid red circles) and $\mathrm{La}_{2} \mathrm{Cu}^{18} \mathrm{O}_{4}$ (open black squares). (a)-(c) Lattice parameters $c, b$, and $a$. (d) In-plane $\mathrm{Cu}-\mathrm{O} 1$ separation, $d_{\mathrm{Cu}-\mathrm{O} 1}$. (e) $\mathrm{Cu}-\mathrm{O} 1-\mathrm{Cu}$ bond angle, $\theta_{\mathrm{Cu}-\mathrm{O} 1-\mathrm{Cu}}$. In addition to structural parameters, these refinements also allow the deduction of an isotropic motional displacement parameter, $B_{\text {iso }}$ (see text), for (f) $\mathrm{Cu}$ ions and (g) in-plane oxygen (O1) ions.

by restricting all of the atoms to isotropic displacements only. The static lattice and atomic parameters are reported in Table I and were found to be completely insensitive to the choice of ionic motion refinement type. The ionic motion parameters extracted from all three types of refinement are summarized in Table II.

We obtain the full temperature dependence of the structural parameters, shown in Fig. 3, from isotropic refinements of our powder-diffraction data at temperatures between 15 and $290 \mathrm{~K}$. We remind the reader that these data were taken with 


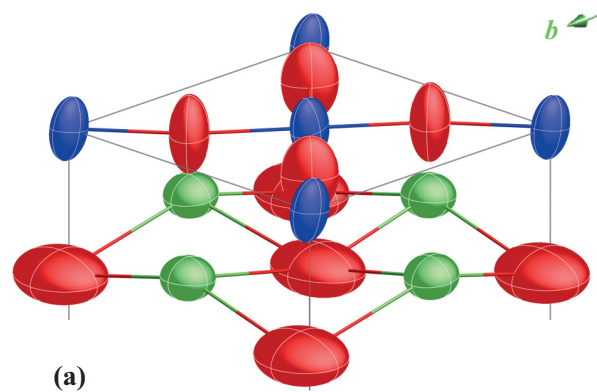

(a)

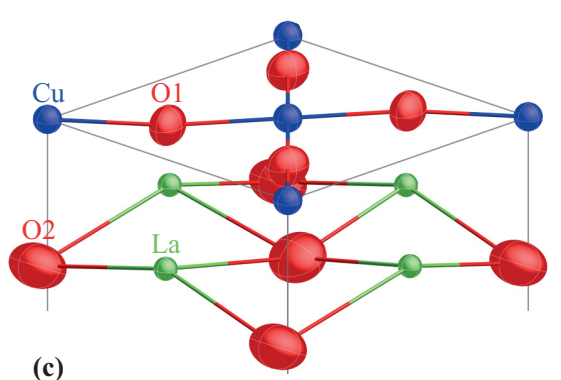

(c)

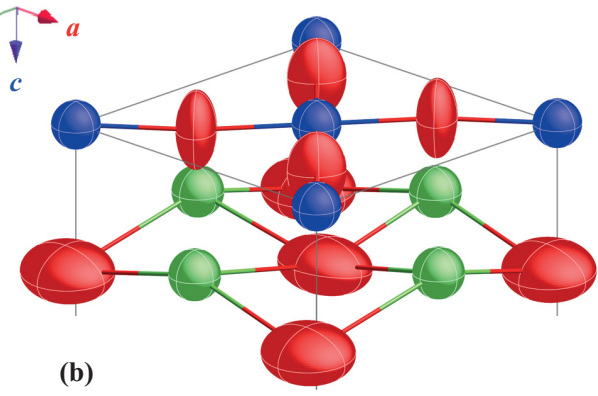

4. (Color online) Crystal structure of $\mathrm{La}_{2} \mathrm{Cu}^{16} \mathrm{O}_{4}$, with the magnitudes of the refined ionic motion parameters illustrated as spheres and ellipsoids. Lattice constants and motional parameters are represented on the same scale. La ions are shown in green, Cu in blue, and $\mathrm{O} 1$ and $\mathrm{O} 2$ in red. (a) Refinement of the $T=290 \mathrm{~K}$ data set with fully anisotropic thermal parameters. (b) Refinement of the $T=290 \mathrm{~K}$ data set performed using anisotropic thermal displacements for the $\mathrm{O} 1$ and $\mathrm{O} 2$ ions but isotropic displacements of $\mathrm{La}$ and $\mathrm{Cu}$ ions. (c) As in (b) for the $T=15 \mathrm{~K}$ data set.

intermediate statistics and therefore the error bars do not match those of Table I. Figures 3(a)-3(c) show the lattice parameters $c, b$, and $a$, whose thermal variation is evidently much larger than the small isotope effect (Sec. III). The $\mathrm{Cu}-\mathrm{O} 1$ bond lengths [Fig. 3(d)] show very little change, indicating that the change in lattice parameters is due primarily to rotation of the $\mathrm{CuO}_{6}$ octahedra of which the structure is composed. This conclusion is reinforced by inspecting the behavior of the $\mathrm{Cu}-\mathrm{O} 1-\mathrm{Cu}$ bond angle [Fig. 3(e)], which is the same for both in-plane bond directions. In Figs. 3(f) and 3(g), we show the isotropic motional displacement parameters for (f) $\mathrm{Cu}$ and (g) $\mathrm{O}$ ions, which we discuss in more detail below. For both ions, it can be seen that the zero-point motion and the thermal motion at $290 \mathrm{~K}$ are of similar magnitude.

Turning to the details of the ionic motion contained in Table II, in Fig. 4 we show the crystal structure with the ionic motion illustrated as spheres (isotropic) and ellipsoids (anisotropic), whose axes are determined by the full width at half maximum (FWHM) height of the ionic motion distribution, expressed by square roots of the parameters $U_{i j}$ in Table II. The isotropic motional parameter shown in Figs. 3(f) and $3(\mathrm{~g})$ is given by $B_{\text {iso }}=8 \pi^{2} U_{\text {iso. }}$. The values of the anisotropic ionic motional parameters, represented by the ellipsoids in Fig. 4, are clearly quite different for in-plane and out-of-plane ions, and also within the planes. The anisotropic deformation of the ellipsoids of ionic motion may be understood by considering the nature of the various bonds in the system. In-plane oxygen atoms are constrained along their bond direction by the presence of $\mathrm{Cu}$ ions on both sides, causing their motion to be primarily perpendicular to the $\mathrm{Cu}-\mathrm{O} 1-\mathrm{Cu}$ bonds. In this plane ( $y z$ for an $x$-axis bond), the restoring forces are lowest for displacements out of the $\mathrm{CuO}_{2}$ planes, and so the ellipsoids are most elongated in the $c$ direction.

The same is true for the $\mathrm{Cu}$ ions, which are relatively tightly

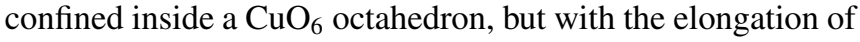
this octahedron along $c$ allowing more motion in this direction. This confinement provides partial justification for the approximation of isotropic displacements necessary to refine the $15 \mathrm{~K}$ data, although of course this does not capture the effects of octahedron elongation. For the out-of-plane La and $\mathrm{O} 2$ ions, the fully anisotropic refinement at $290 \mathrm{~K}$ (Fig. 4) shows that their in-plane motion is stronger, and is relatively isotropic due to the lack of confining atoms in this structural layer.
For all of the atoms, zero-point fluctuations account quite uniformly for much more than half of the net ionic motion at $290 \mathrm{~K}$ (Fig. 4). This result is quantified for the in-plane ions in Fig. 5, which compares the ionic motion of the $\mathrm{Cu}$ and $\mathrm{O} 1$ ions at 15 and $290 \mathrm{~K}$, and is in close quantitative agreement with a straightforward Debye model for phonons in cuprates [24]. If the zeropoint motion has a characteristic root-mean-square displacement $\sqrt{\left\langle u^{2}\right\rangle}=A$, then for temperatures above the Debye temperature $\left(T>\Theta_{D}\right)$, one has $\left\langle u^{2}(T)\right\rangle=4 A^{2} T / \Theta_{D}$ [25]. Using, for the region $T<\Theta_{D}$, the approximate interpolation formula $\sqrt{\left\langle u^{2}(T)\right\rangle}=A \sqrt{1+4 T / \Theta_{D}}$, returns $\sqrt{\left\langle u^{2}(T=290 \mathrm{~K})\right\rangle} / \sqrt{\left\langle u^{2}(T=15 \mathrm{~K})\right\rangle}=1.9$ for cuprate materials, where $\Theta_{D}=450 \mathrm{~K}$. At $15 \mathrm{~K}$, we find that the zero-point motion of $\mathrm{O} 1$ is almost isotropic in the $y$ and $z$ directions, but the motion along $z$ grows significantly with temperature, causing the distortion of the motional ellipsoids we observe at $290 \mathrm{~K}$. As a consequence, the leading effect of thermal motion on the $\mathrm{Cu}-\mathrm{O} 1-\mathrm{Cu}$ bonds is on the bond angle [Fig. 3(e)]. In fact this observation explains the asymmetric distribution of

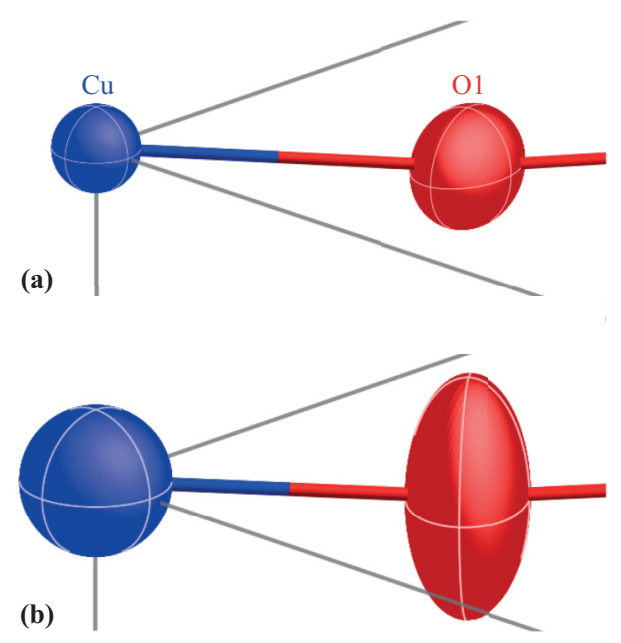

FIG. 5. (Color online) Zero-point and thermal contributions to ionic motion, compared using the parameters for $\mathrm{Cu}$ (blue) and $\mathrm{O} 1$ (red) at (a) $15 \mathrm{~K}$ and (b) $290 \mathrm{~K}$, given by the half-anisotropic refinement. 
magnetic interaction strengths at high temperature, which we will discuss in Sec. IV.

\section{OXYGEN ISOTOPE EFFECT}

We express the OIE on a quantity $x$ by $\Delta x=\frac{x^{18}-x^{16}}{x^{16}}$, where $x^{16}$ and $x^{18}$ are shorthand for the quantity $x$ measured, respectively, in $\mathrm{La}_{2} \mathrm{Cu}^{16} \mathrm{O}_{4}$ and $\mathrm{La}_{2} \mathrm{Cu}^{18} \mathrm{O}_{4}$. Our ${ }^{18} \mathrm{O}$ samples are not pure $\mathrm{La}_{2} \mathrm{Cu}^{18} \mathrm{O}_{4}$, but $78 \%$ substituted. While complete isotopic substitution is difficult to achieve, the OIE on most of the quantities we measure turns out to be very small, and thus it is reasonable to assume that it is linear in the degree of isotopic substitution. Thus, to a good approximation, the true OIE, in the event that $100 \%$ substitution could be achieved, would be $100 / 78$, or approximately 1.25 times as large as the effects we observe.

We begin our analysis of the OIE in $\mathrm{La}_{2} \mathrm{CuO}_{4}$ by considering the susceptibility (Fig. 1). Oxygen isotope substitution raises the susceptibility peak by $2.4 \pm 0.2 \mathrm{~K}$, and hence the OIE $\Delta T_{N}=0.92 \pm 0.07 \%$ is positive. While this result contradicts that reported in Ref. [16], we emphasize that these authors found their values of $T_{N}$, and thus also of the OIE on $T_{N}$, to depend strongly on the preparation and annealing conditions of their samples.

Turning to the structural analysis, the OIE on the ionic position and motion can be extracted from the information in Table II. The OIEs obtained for the lattice parameters $a, b$, and $c$, the $\mathrm{Cu}-\mathrm{O} 1$ bond length, the $\mathrm{Cu}-\mathrm{O} 1-\mathrm{Cu}$ bond angle, and the isotropic ionic motion parameters at 15 and at $290 \mathrm{~K}$ are summarized in Table III. The temperature dependence of each of these parameters is shown in Fig. 3.

The OIE on the crystal lattice corresponds to a very small overall contraction of order $10^{-4}$. The negative OIE on the $c$-axis lattice parameter, which is expected to increase the weak interactions in this direction, agrees qualitatively with the positive OIE we measured for the magnetic ordering temperature $T_{N}$. The OIE on the $\mathrm{Cu}-\mathrm{O} 1$ bond length is also negative, which will have a minor effect on the in-plane interaction parameters we discuss in Sec. IV. The OIE on the static bond angle is entirely negligible.

However, the OIE on the ionic motion is significant, ranging from $-23 \%$ to $+50 \%$ in some parameters. The motional ellipsoids of all the ions for $\mathrm{La}_{2} \mathrm{Cu}^{16} \mathrm{O}_{4}$ and $\mathrm{La}_{2} \mathrm{Cu}^{18} \mathrm{O}_{4}$ are compared in Fig. 6. At low temperatures, a careful inspection of the half-anisotropic refinements for the two samples [Figs. 6(a) and 6(b)] shows a reduction, or negative OIE, of the order of $20 \%$ in zero-point $\mathrm{O} 1$ and $\mathrm{O} 2$ ionic motion from ${ }^{16} \mathrm{O}$ to ${ }^{18} \mathrm{O}$, and that this is accompanied by a similar positive OIE in $\mathrm{Cu}$ and La motion (Table III). At $290 \mathrm{~K}$, the fully anisotropic refinements in Figs. 6(c) and 6(d) show that the thermal contributions to ionic motion are similar for both ${ }^{16} \mathrm{O}$ to ${ }^{18} \mathrm{O}$, effectively suppressing the motional OIEs to values of the order of $10 \%$ (Table III). The increasing thermal motion is also quite anisotropic, displaying strong increases in the $c$-axis motion of the in-plane $\mathrm{Cu}$ and $\mathrm{O} 1$ ions and a corresponding flattening in the ellipsoids of the out-of-plane $\mathrm{La}$ and $\mathrm{O} 2$ ions. These results are qualitatively consistent with expectations from a simple ball-and-spring model for the structure of anisotropic $\mathrm{CuO}_{6}$ octahedra and with the sum
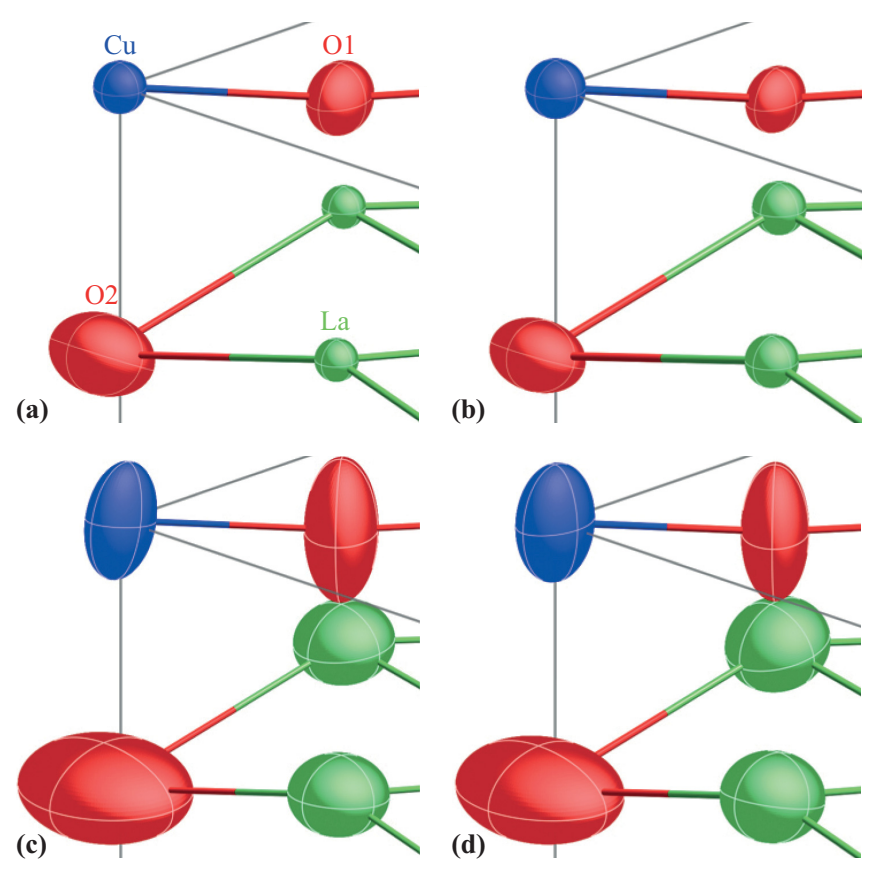

FIG. 6. (Color online) Oxygen isotope effect on the ionic motion parameters from comparison of (a), (c) $\mathrm{La}_{2} \mathrm{Cu}^{16} \mathrm{O}_{4}$ and (b),(d) $\mathrm{La}_{2} \mathrm{Cu}^{18} \mathrm{O}_{4}$. (a),(b) Results from the half-anisotropic structural refinement at $15 \mathrm{~K}$ show a reduction in $\mathrm{O} 1$ and $\mathrm{O} 2$ motion leading to a corresponding increase in $\mathrm{Cu}$ and La motion. (c),(d) Results from the fully anisotropic structural refinement at $290 \mathrm{~K}$ show that thermal fluctuations suppress the OIE on the motional parameters while increasing their $c$-axis anisotropy.

rule for lattice vibrations [26-28]. Isotopic substitution also has a small effect on the directions of ionic motion, which can be seen in the orientation of the ellipsoids in Figs. 6(c) and $6(d)$.

\section{MOTIONAL RENORMALIZATION OF ELECTRONIC AND MAGNETIC PROPERTIES}

Changes in ionic positions have a natural effect in altering the electronic and magnetic coupling constants. In the effective one-band, strong-coupling model for the behavior of the doped cuprate plane in $\mathrm{La}_{2} \mathrm{CuO}_{4}$, these are denoted as $t$ for the hopping of holelike quasiparticles and $J$ for the antiferromagnetic superexchange interaction between $S=1 / 2$ spins [29]. Here we focus primarily on $J$ and estimate the effects of ionic motion by following one detailed theoretical analysis of a single cuprate plane [30].

The energy of thermal motion in $\mathrm{La}_{2} \mathrm{CuO}_{4}$ lies largely in the range 10-20 meV [31], while the bandwidth of antiferromagnetic exchange processes extends up to $300 \mathrm{meV}$ [4], corresponding to $J \simeq 140 \mathrm{meV}$. Thus, the ionic displacement is slow compared to the time scale (inverse energy scale) of the electronic parameters and the Born approximation may be justified. Here we comment that an alternative means of incorporating lattice effects on the electronic and magnetic properties would be a first-principles lattice dynamics calculation for the complete phonon spectrum [32]. From this, one may determine the specific phonons that are most important for particular electronic coupling effects; these are usually thought 
to be the "breathing modes" of the cuprate squares, involving longitudinal motion of $\mathrm{Cu}$ and $\mathrm{O}$ ions along their bonding axes. However, phonons relevant for this type of process, which do have significant effects on the quasiparticle properties, usually lie in the 50-80 meV region [33] and are not important at room temperature.

\section{A. Estimation of superexchange parameters}

The effects of temperature on magnetic interactions have been considered in a general microscopic framework [34], where the dominant behavior is a sharp fall in $J(T)$ due to thermal expansion of the system. This effect, which in some systems is large enough to be observable in the susceptibility peak position, is contained in our thermal data but turns out to be weak. We begin a more specific microscopic analysis by considering the integral describing the overlap of $\mathrm{Cu} d$ orbitals and $\mathrm{O} 1 p$ orbitals. From the general theory of orbital overlap [35],

$$
t_{p d}=A_{0} d^{-\alpha_{0}} \cos ^{\beta_{0}} \theta,
$$

where $d=\left|\mathbf{r}_{\mathrm{Cu}}-\mathbf{r}_{\mathrm{O}}\right|$ is the spatial separation of the $\mathrm{Cu}$ and $\mathrm{O}$ ions and $\theta$ describes their angular deviation away from the situation of a perfect $\sigma$-orbital alignment; thus, $d \equiv d_{\mathrm{Cu}-\mathrm{O} 1}$ and $\theta \equiv \theta_{\mathrm{Cu}-\mathrm{O} 1-\mathrm{Cu}}$ are precisely the structural parameters of Sec. II. $A_{0}$ is a constant of proportionality, and the power-law dependences in $d$ and $\cos \theta$ are given, respectively, by $\alpha_{0}=3$ or 3.5 and $\beta_{0}=1$.

In the cuprate geometry, the lowest-order processes in a perturbative expansion for the quasiparticle hopping and superexchange interaction yield the parameter dependences $t \propto t_{p d}^{2}$ (second order) and $J \propto t_{p d}^{4}$ (fourth order). However, the cuprate plane has long been known to be very poorly described by lowest-order perturbative approaches, and this has led to detailed efforts to derive more accurate low-energy descriptions from $d-p$ [36] or three-band [37] models for the $\mathrm{Cu}$ and $\mathrm{O}$ orbitals. For clarity, we follow the systematic extension of the lowest-order analysis [30], where the direct in-plane O-O hopping, described by the overlap integral $t_{p p}$, contributes to $J$ through many possible fifth- and sixthorder processes. We take account of these contributions by computing the effective overlap integral,

$$
J=A\left(d_{1} d_{2}\right)^{-\alpha / 2}\left(\cos \theta_{1} \cos \theta_{2}\right)^{\beta / 2},
$$

for a given bond $\mathrm{Cu}(1)-\mathrm{O}-\mathrm{Cu}(2)$. Here, $d_{1}=\left|\mathbf{r}_{\mathrm{Cu}(1)}-\mathbf{r}_{\mathrm{O}}\right|$ and $d_{2}=\left|\mathbf{r}_{\mathrm{Cu}(2)}-\mathbf{r}_{\mathrm{O}}\right|$ are the respective separations of the two $\mathrm{Cu}$ ions from the same $\mathrm{O}$ ion, while $\theta_{1}$ and $\theta_{2}$ are the corresponding bonding angles and $\alpha$ and $\beta$ denote effective power-law dependences. By considering only the lowest-order contribution to $J$, one would expect the very strong powers $\alpha \simeq$ $12-14$ and $\beta=4$. However, the extensive contributions from higher-order terms [30], primarily at the fifth and sixth order in $t_{p d}$ and $t_{p p}$, reduce the effective powers to values around $\alpha=7$ and $\beta=2$. These are the values emerging directly from cell-perturbation models of the cuprate plane [36,37], which attempt to construct a perturbation theory based on the hybridized $\mathrm{CuO}_{4}$ unit and deduce that $J \propto t_{p d}^{2}$. The best theoretical estimate provided by Ref. [30] was $J \propto d^{-6.9}$, which was in good agreement with an experimental estimate $J \propto d^{-6.4}$ deduced [38] from a high-pressure study.
Here we use the form of Eq. (2) in combination with a statistical distribution of $\mathrm{Cu}$ and $\mathrm{O}$ positions, whose probabilities are determined from the experimental measurements represented in Fig. 4. Mindful of the fact that the $\mathrm{CuO}_{2}$ lattice contains two $\mathrm{O}$ atoms for each $\mathrm{Cu}$, we calculate superexchange parameters not for a single bond (two $\mathrm{Cu}$ and one $\mathrm{O}$ atom), but for a small lattice in order to represent appropriately the weight of each probabilistic function. Our primary analysis of the resulting data is simply to take a histogram for the probability of finding a given bond strength, which takes into account all of the correlations between the positions of two ions in one bond, including the thermal-expansion effect [34]. However, a secondary effect is that the motion of one $\mathrm{Cu}$ ion clearly induces correlations among all the neighboring bonds (specifically, $\langle i-x, i\rangle,\langle i, i+x\rangle,\langle i-y, i\rangle$, and $\langle i, i+y\rangle$ ), and we comment on this point below.

\section{B. Motional renormalization}

In Fig. 7(a), we show the distribution functions obtained for the superexchange parameter $J$ in $\mathrm{La}_{2} \mathrm{Cu}^{16} \mathrm{O}_{4}$ at the two experimental temperatures ( $T=15$ and $290 \mathrm{~K}$ ), and in Fig. 7(b),
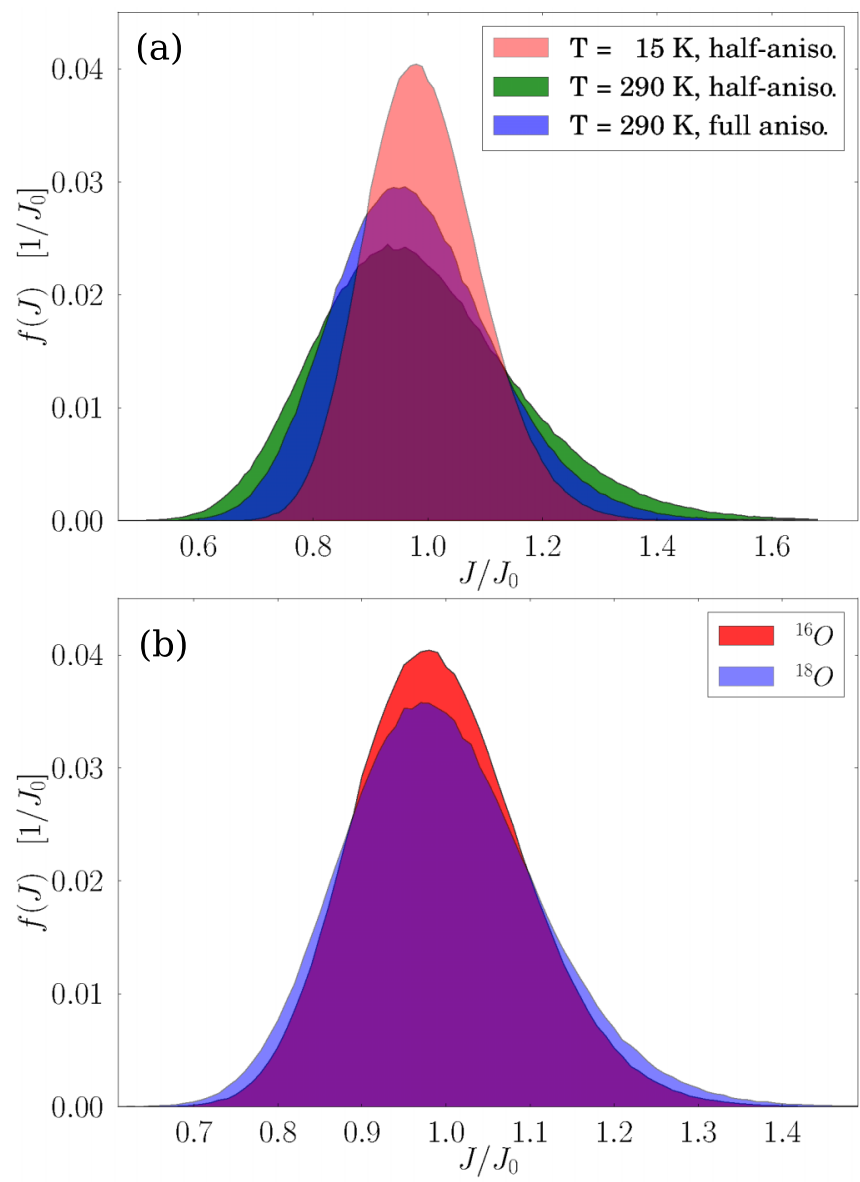

FIG. 7. (Color online) Distribution histogram of the superexchange parameter $J$, normalized to its uniform-lattice value $J_{0}$ (Table IV), due to zero-point and thermal $\mathrm{Cu}$ and $\mathrm{O}$ ionic motion. (a) Histograms for the three structural refinements of $\mathrm{La}_{2} \mathrm{Cu}^{16} \mathrm{O}_{4}$. (b) Histograms comparing $J$ for $\mathrm{La}_{2} \mathrm{Cu}^{16} \mathrm{O}_{4}$ and $\mathrm{La}_{2} \mathrm{Cu}^{18} \mathrm{O}_{4}$ at $15 \mathrm{~K}$ with half-anisotropic motional parameters. 
TABLE IV. Analysis of superexchange parameters determined from the ionic motion contained in the six structural refinements. The "static" value $J_{0}$ is calculated for the ionic positions at the centers of the distributions and is normalized to the value $J_{0}^{r}$ for $\mathrm{La}_{2} \mathrm{Cu}^{16} \mathrm{O}_{4}$ at $15 \mathrm{~K}$. The average superexchange value $\langle J\rangle$ is taken over the whole distribution. The most likely value is denoted as $J_{\mathrm{m}}$. The standard deviation of the distribution is specified by $\Delta J$, with the FWHM given by $2.35 \Delta \mathrm{J}$.

\begin{tabular}{lcccc}
\hline \hline & $J_{0} / J_{0}^{r}$ & $\langle J\rangle / J_{0}^{r}$ & $J_{\mathrm{m}} / J_{0}^{r}$ & $\Delta J / J_{0}^{r}$ \\
\hline${ }^{16} \mathrm{O} 15$ K half-anis. & 1.000 & 0.999 & 0.984 & 0.099 \\
${ }^{18} \mathrm{O} 15$ K half-anis. & 1.001 & 1.002 & 0.982 & 0.111 \\
${ }^{16} \mathrm{O} 290$ K half-anis. & 0.995 & 0.992 & 0.947 & 0.163 \\
${ }^{18} \mathrm{O} 290 \mathrm{~K}$ half-anis. & 0.996 & 0.995 & 0.945 & 0.171 \\
${ }^{16} \mathrm{O} 290 \mathrm{~K}$ full anis. & 0.995 & 0.983 & 0.956 & 0.133 \\
${ }^{18} \mathrm{O} 290 \mathrm{~K}$ full anis. & 0.996 & 0.987 & 0.953 & 0.144 \\
\hline \hline
\end{tabular}

we compare the distributions for $\mathrm{La}_{2} \mathrm{Cu}^{16} \mathrm{O}_{4}$ and $\mathrm{La}_{2} \mathrm{Cu}^{18} \mathrm{O}_{4}$ at $15 \mathrm{~K}$. Qualitatively, it is clear that there is a significant distribution of $J$ values even at low temperature. The increase in width of the distribution from 15 to $290 \mathrm{~K}$ is a consequence of the increase in $U$ values shown in Table II and Fig. 4, and will be quantified below. The width of the distribution at $290 \mathrm{~K}$ differs slightly between the half-anisotropic and fully anisotropic refinements, but the qualitative features are the same [Fig. 7(a)]. The distribution is slightly wider for $\mathrm{La}_{2} \mathrm{Cu}^{18} \mathrm{O}_{4}$ than for $\mathrm{La}_{2} \mathrm{Cu}^{16} \mathrm{O}_{4}$ [Fig. 7(b)], indicating that the effects on $J$ of the reduced $\mathrm{O}$ ion motion [Fig. 6] are more than compensated for by the additional $\mathrm{Cu}$ ion motion this allows. It is also evident that the distributions are asymmetric, with the maximum value $J_{\mathrm{m}}$ shifting down in energy but a longer tail extending towards higher values of $J$. We discuss the possible consequences of such a distribution for the magnetic and also the electronic properties of the cuprates below.

First we consider the properties of the histograms we compute. Table IV characterizes the histograms for the six different structural refinements by their mean, their peak position (corresponding to the most likely value of $J$ ), and their standard deviation. The parameter $J_{0}$ is the superexchange interaction expected if the ions are stationary and located in their conventional atomic positions. All of the values quoted for $\langle J\rangle, J_{\mathrm{m}}$, and $\Delta J$ in Table IV are normalized to $J_{0}^{r}$, calculated for $\mathrm{La}_{2}{ }^{16} \mathrm{CuO}_{4}$ at $15 \mathrm{~K}$.

The variation we compute in the static value $J_{0}$ is as expected. It is marginally higher for ${ }^{18} \mathrm{O}$ due to the slight contraction of the lattice, and marginally lower at $290 \mathrm{~K}$ due to the thermal expansion of the lattice. The variations are, however, tiny and the $0.1 \%$ OIE is insufficient to account for the $0.9 \%$ increase in $T_{N}$. Including the effects of ionic motion leads to a variation larger by a factor of 3-4 as a function of isotope and temperature, and the $0.4 \%$ OIE on $\langle J\rangle$ is a much more significant factor in explaining the change in $T_{N}$. It should be noted that $T_{N}$ is a combination of the in-plane correlation length $\xi$, which is controlled by $J$ and also increases exponentially with decreasing temperature [39], and the small interlayer coupling and effective spin-space anisotropies, which are more difficult to estimate. We return to these topics below.

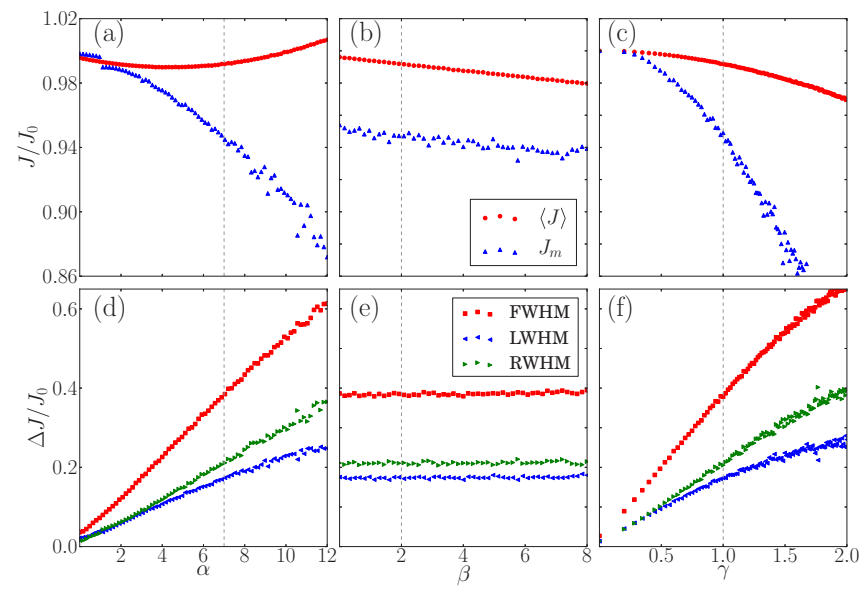

FIG. 8. (Color online) Variation of the superexchange parameter $(J)$ distribution with (a),(d) the exponent $\alpha$ governing the dependence on ionic separation $d$ [Eq. (1)], (b),(e) the exponent $\beta$ governing the dependence on relative orbital orientation $\theta$ [Eq. (1)], and (c),(f) the scale factor $\gamma$ applied to the ionic motion distribution (an analog of the temperature). (a)-(c) The average $\langle J\rangle$ and peak value $J_{\mathrm{m}}$. (d)-(f) The left-width, right-width, and full-width at half maximum $\Delta J$ of the distribution.

The most remarkable result of our study is unquestionably the significant standard deviation of the distribution, which we find to vary between 10 and $20 \%$ for realistic parameters in Eq. (2). Further, while the OIE on the average value $\langle J\rangle$ is only $0.4 \%$, the widths of the $J$ distributions are $8-12 \%$ larger for ${ }^{18} \mathrm{O}$ than for ${ }^{16} \mathrm{O}$. Experimental observables sensitive to this width could therefore show a significant OIE. The standard deviation $\Delta J$ quantifies the observation, made in Fig. 7(a), that the width of the $J$ distribution increases by a factor of approximately 1.6 on increasing the temperature from 15 to $290 \mathrm{~K}$. This indicates that thermal fluctuations contribute rather less to the effect of ionic motion than do the zero-point fluctuations even at room temperature, and thus that our considerations are important at all temperatures in cuprates. We comment also that the width of the distribution resulting from the fully anisotropic structural refinement is significantly smaller than for the half-anisotropic one, and we expect that this is a consequence of the enhanced $z$-axis $\mathrm{Cu}$ motion in the former fit (Table II) appearing as in-plane motion in the latter.

Considering briefly the functional dependence of the superexchange distribution, the average changes little while the peak value falls significantly with the power $\alpha$ governing (2) the ionic separation [Fig. 8(a)], whereas both fall only weakly with the power $\beta$ governing the angle [Fig. 8(b)]. By contrast, the standard deviation increases linearly with $\alpha$ [Fig. 8(d)] but is little affected by $\beta$ [Fig. 8(e)]. These results are fully in line with physical expectations, contained in an expansion in small $\left\langle u^{2}\right\rangle$ : the mean behaves quadratically in $\alpha$ and linearly in $\beta$, while the standard deviation rises linearly with $\alpha$ but depends only weakly on $\beta$ because the approximately $180^{\circ}$ static angle of the $\mathrm{Cu}-\mathrm{O}-\mathrm{Cu}$ bond makes the leading contribution of an angular deviation very small. In evaluating the mean and standard deviation for the distributions in Table IV, we have taken the values $\alpha=7$ and $\beta=2$ (Sec. IV A). While we do not have the experimental data to analyze all temperatures, 
the effect of increased thermal ionic motion may be simulated approximately by applying a scale factor to the ionic motion parameters. Again, the average $\langle J\rangle$ and peak value $J_{\mathrm{m}}$ decrease quadratically with the amplitudes of ionic motion [Fig. 8(c)], while the standard deviation $\Delta J$ increases linearly before rounding off towards $\gamma=2$ [Fig. 8(f)]. This last result explains why the near doubling of ionic motion amplitudes between $15 \mathrm{~K}$ and room temperature (Table II) leads only to a $60 \%$ broadening in the distribution of $J$ values (Table IV).

\section{Consequences of motional renormalization}

We turn now to a discussion of the effects on the electronic and magnetic properties in the cuprate materials of the fact that $t$ and $J$ do not have fixed values. Instead, both parameters obey a fluctuating distribution of values whose mean drops slightly with increasing temperature due to the thermal expansion of the lattice [34], and whose functional form is an approximately Gaussian distribution where the width is determined by both zero-point and thermal fluctuations. The width of the distribution is significant even at the lowest temperatures, spanning a range of the order of $30 \mathrm{meV}$ (Fig. 7). While the thermal expansion is, from our data, small in cuprates and thus has little effect on the mean value $\langle J\rangle$, the broadening $\Delta J$ may have important effects on thermodynamic measurements, such as the susceptibility, and on dynamical measurements, such as the optical response and the magnon spectrum. These latter effects can be expected primarily in the widths of the excited modes, rather than in their positions, but may also induce interactions between excitations.

A key question is how ionic motion may affect the Néel temperature. Our analysis gives only a partial impression of thermally induced shifts in $T_{N}$ because this quantity is critically dependent on both the $z$-axis coupling and the effective $X Y$ anisotropy arising from weak DzyaloshinskiiMoriya interactions in the plane, and the ionic motion effect on these terms in the Hamiltonian cannot be estimated accurately from our data. For doped cuprates, one of the most important sets of static and dynamical effects arising from motional broadening of $t$ and $J$ will be on superconductivity itself. Here, only our zero-point motional results are relevant, and their effects can include changes to $T_{c}$, to the gap $\Delta(\mathbf{k})$, and also to the origin of an isotope effect. While $T_{c}$ is a consequence of three-dimensional coupling, and thus is subject to some of the same factors as $T_{N}, \Delta(\mathbf{k})$ is a largely in-plane quantity that is thought in many theories to depend linearly on $J$, and would therefore be reduced and broadened in quantities such as the quasiparticle dispersion. Our experimental results show a very weak OIE on the average in-plane superexchange interaction. However, motional broadening is $8-12 \%$ larger in the ${ }^{18} \mathrm{O}$ system than in the ${ }^{16} \mathrm{O}$ material, and this will affect the quasiparticle properties. Thus our results suggest that the origin of the isotope effects measured for a variety of static and dynamic quantities in cuprates [40], including the infra-red optical response [41] and the photoemission spectrum [42], lies in the $z$-axis coupling (above) for static quantities and in the nature of the quasiparticles for dynamical ones.

To quantify some of the static and dynamical consequences of motional modulation of the magnetic interaction, we performed a series of quantum Monte Carlo (QMC) calculations. By using the motional parameters for $\mathrm{La}_{2} \mathrm{Cu}^{16} \mathrm{O}_{4}$ at 15 and $290 \mathrm{~K}$, we generated lattices with a distribution of coupling values to represent snapshots of the ionic motion. The staggered magnetization in the ground state was then determined by finite-size extrapolation of $L \times L$ lattices using 10 realizations of the ionic positions for $L=16,32$, and 64 and five realizations for $L=128$. The result for uniform couplings, $m_{s}=0.3072 \pm 0.0007$, is in good agreement with the literature [3]. Our result for the lattice of interaction strengths modulated by the positional distribution at $15 \mathrm{~K}$ is $m_{s}=0.3045 \pm 0.0002$, a reduction of the order $(\Delta J / J)^{2} \approx$ $1 \%$ expected from mean-field theory for weak $\Delta J$ (we recall that $m_{s}$ is independent of $\langle J\rangle$ itself) [24].

Further examples of static or slowly varying quantities in which ionic motion effects may be appreciable include the correlation length $\xi(T)$ and the temperature dependence of the instantaneous structure factor $S(\mathbf{q}=0, T)$. Both quantities are amenable to numerical simulation, and temperature-dependent data exist for $\xi(T)$ up to $800 \mathrm{~K}$ (a value mandating a more sophisticated treatment of the phonon spectrum). While the extent to which a frozen-distribution approach can correctly capture the effects of ionic motion remains an open question, we expect that with adequate disorder averaging, it is appropriate for static or slowly varying properties such as $m_{s}, \xi$, and $S(\mathbf{q}=0)$ at low temperatures; our simulations at 15 and $290 \mathrm{~K}$ are for weak thermal disorder, where all realizations return very similar results for the system sizes employed.

As an example of a dynamical quantity, we have investigated the consequences of ionic motion for the highenergy spin excitations in $\mathrm{La}_{2} \mathrm{CuO}_{4}$, which occur around $300 \mathrm{meV}$. Because this energy lies well above all the phonon frequencies (antiadiabatic limit), motional effects on the spin response should once again be independent of any possible strong coupling to specific phonon modes, justifying the incoherent approximation and a frozen-distribution calculation. We focus on the antiferromagnetic zone-boundary point, $\mathbf{q}=(\pi / 2, \pi / 2)$, in order to separate motional fluctuation effects from the intrinsic quantum effects observed around the $(\pi, 0)$ point in 2D Heisenberg antiferromagnets [43-45]. We calculated the transverse dynamical structure factor on a $16 \times 16$ lattice with periodic boundary conditions, using the stochastic series-expansion quantum Monte Carlo technique with directed loop updates [46]. To determine the transverse component, a small field $h_{z}=0.01 J_{0}$ was imposed to break the spin-rotational symmetry. The dynamical quantities were extracted from the imaginary-time Monte Carlo data using a stochastic analytic continuation technique $[47,48]$.

Figure 9(a) compares the temperature dependence of the line shape obtained for a homogeneous lattice, meaning with constant values $J=J_{0}$ on every bond, to that obtained using $J$ values distributed according to the motional parameters we have deduced for $\mathrm{La}_{2} \mathrm{Cu}^{16} \mathrm{O}_{4}$ at $15 \mathrm{~K}\left(k_{B} T \simeq 0.01 J_{0}\right)$ and at $290 \mathrm{~K}\left(k_{B} T \simeq 0.2 J_{0}\right)$. We characterize the line shape by the linewidth $\Gamma$, which we deduce from the half width at half maximum (HWHM) height on the low-energy side of the peak in the dynamical structure factor, because the high-energy side of the spectrum is extended by continuum states. The finite linewidth $\Gamma_{i}=0.051 J$, for a constant $J$ at low temperatures, is a consequence of these higher-energy continua combined with the limitations inherent in analytic continuation of QMC 

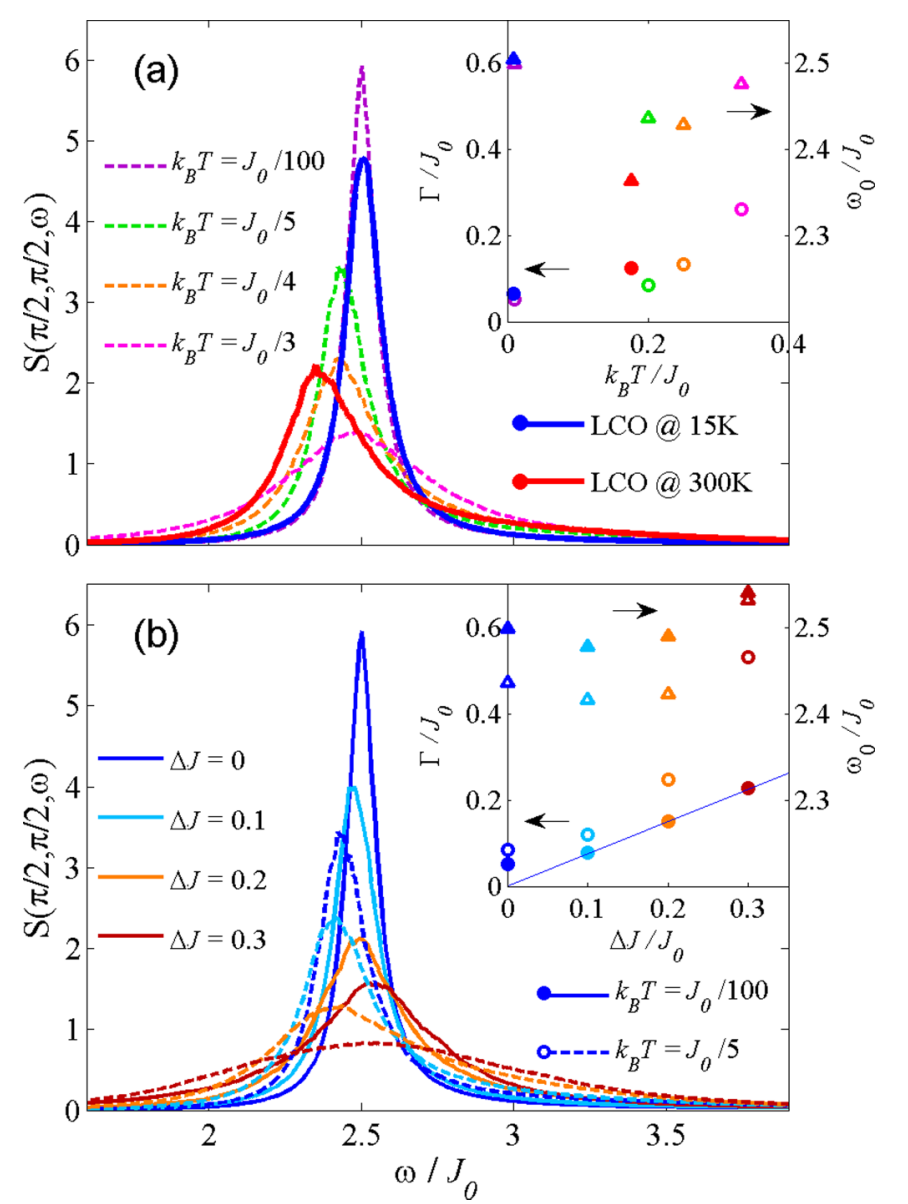

FIG. 9. (Color online) Energy dependence of the transverse dynamical structure factor $S(\mathbf{q}, \omega)$ at $\mathbf{q}=(\pi / 2, \pi / 2)$, computed by quantum Monte Carlo simulations. (a) Calculations for $\mathrm{La}_{2} \mathrm{CuO}_{4}$ using the $J$ distributions appropriate for the measured ionic motion at 15 and $290 \mathrm{~K}$ (solid lines). For comparison, we show as dashed lines the results expected from a purely thermal broadening, i.e., with constant $J=J_{0}$, for chosen values of $J_{0} / k_{B} T$. Inset: temperature dependence of peak energy $\omega_{0} / J_{0}$ (triangles) and peak width $\Gamma / J_{0}$ (circles) for $\mathrm{La}_{2} \mathrm{CuO}_{4}$ at both experimental temperatures (solid symbols) and for uniform $J$ with thermal broadening (open symbols). (b) Calculations made using Gaussian distributions of $J$ values with increasing standard deviations $\Delta J$ for $k_{B} T=J_{0} / 100$ (solid lines) and for $k_{B} T=J_{0} / 5$ (dashed lines). Inset: dependence of $\Gamma$ (circles) and $\omega_{0}$ (triangles) for $k_{B} T=J_{0} / 100$ (solid symbols) and for $k_{B} T=J_{0} / 5$ (open symbols). The line indicates the linear relationship $\Gamma=0.75 \Delta J$ for $k_{B} T=J_{0} / 100$.

data [49,50]. However, we will show below that the majority of the broadening we calculate for $\mathrm{La}_{2} \mathrm{CuO}_{4}$ at $15 \mathrm{~K}, \Gamma=0.064 \mathrm{~J}$, is in fact a consequence of the zero-point fluctuations in $J$ and is not due to this intrinsic QMC linewidth. On raising the temperature, we find that the combined effects of thermal spin fluctuations and ionic motion lead to a very strong broadening $\Gamma=0.124 J$ at $290 \mathrm{~K}$, which is $50 \%$ wider than the value $\Gamma=0.084 J$ obtained for the uniform lattice at $k_{B} T=0.2 J_{0}$ [Fig. 9(a)].

To further elucidate the effect of a distribution of $J$ values on the excitation linewidth, and of how this effect combines with thermal spin fluctuations, we show in Fig. 9(b) calculations performed at $k_{B} T=J_{0} / 100$ and $k_{B} T=J_{0} / 5$ using Gaussian distributions of $J$ with standard deviations from 0 to $0.3 J_{0}$. At low temperature, this effective fluctuation of $J$ causes a linear increase in the excitation linewidth, $\Gamma=0.75 \Delta J$ [inset, Fig. 9(b)]. This is the observation allowing us to disentangle a broadening of $S(\mathbf{q}, \omega)$ induced by ionic motion ( $J$ fluctuation) from the intrinsic low-temperature QMC linewidth, $\Gamma_{i}$, computed with uniform $J$ values. Indeed, taking $\Delta J=0.099 J_{0}^{r}$ at $15 \mathrm{~K}$ from Table IV, a linear interpolation yields $\Gamma=0.074 \mathrm{~J}$, which is a value close to that simulated directly for $\mathrm{La}_{2} \mathrm{CuO}_{4}$ at this temperature. These results demonstrate that this calculated linewidth arises primarily from ionic motion, reflecting little or no influence from $\Gamma_{i}$ when $\Gamma>\Gamma_{i}$. Adding thermal spin fluctuations corresponding to $k_{B} T=J_{0} / 5$ has only a small effect on the uniform- $J$ lattice, but strongly enhances the broadening on the distributed- $J$ lattices. By contrast, the effect of thermal fluctuations on the peak position seems to be only an additive constant; fluctuations of $J$ lead to rather small shifts in peak position, with a minimum around $\Delta J \sim 0.15 J$, which happens to correspond to $\mathrm{La}_{2} \mathrm{CuO}_{4}$ at $290 \mathrm{~K}$, while the softening caused by thermal spin fluctuations is almost constant $(2.5 \%)$ for $0 \leqslant \Delta J \leqslant 0.2 J_{0}$. The width and apparent hardening we find for $\Delta J=0.3 J_{0}$ at $k_{B} T=J_{0} / 5$ is probably the result of the broadening magnon peak merging with the higher-energy continuum in the analytic continuation.

From these results, we may conclude that the distribution of $J$ values at $15 \mathrm{~K}$ causes a line broadening of the order of $0.12 J$ in the FWHM, which is approximately $5 \%$ of the spin-wave energy at $\mathbf{q}=(\pi / 2, \pi / 2)$. At $290 \mathrm{~K}$, this broadening doubles to a FWHM of order $10 \%$ of the spin-wave energy, accompanied by a sizable softening (below). These levels of broadening should be readily detectable at time-of-flight neutron spectrometers such as MAPS at the ISIS spallation source (U.K.), which can reach 2-5\% energy resolutions (FWHM) by employing higher-resolution instrument configurations than those used in previous experiments $[4,51]$. We also draw attention to the fact that ionic motion leads to significantly larger softening of the peak position $(5.5 \%$ at $290 \mathrm{~K})$ than do thermal spin fluctuations alone (2.5\%). Indeed, the values reported [4] for $\mathrm{La}_{2} \mathrm{CuO}_{4}$, $J=146.3 \mathrm{meV}$ at $10 \mathrm{~K}$ and $J=138.3 \mathrm{meV}$ at $300 \mathrm{~K}$, differ by exactly $5.5 \%$. However, a more detailed analysis taking into account the further-neighbor interactions $[52,53]$ would be required for such a comparison to be conclusive.

A further example of a physical observable that we anticipate may reflect strong motional effects is the " $3 J$ " peak observed by two-magnon Raman scattering in cuprate systems [54]. While linear spin-wave theory predicts a sharp twomagnon peak at $\hbar \omega=4 J$, experiments on undoped cuprates, including $\mathrm{La}_{2} \mathrm{CuO}_{4}$, display an anomalously broad (FWHM $\Gamma_{2 m}>J$ ) and asymmetric peak centered around $3 J$ [55]. Elements contributing to the lower energy and broadening of the Raman peak include magnon-magnon interactions [56], strong additional exchange terms within a three-band model [57], and further-neighbor hopping terms [53]. While ionic motion effects will provide a significant broadening even at lowest order in the spin-wave treatment, our results suggest that this broadening would not exceed $30 \%$ of $J_{0}$ even at elevated temperatures. Thus ionic motion is unlikely to provide a full account of the full $3 J$ peak broadening observed in cuprates, and a quantitative explanation of the Raman spectrum would 
have to address the combination of all of the above phenomena. A further example of strong effects arising when magnetic couplings are subject to a local modulation was presented recently [58] in a combined theoretical and experimental study of the lifetime of gapped excitations in the 2D $X Y$ antiferromagnet $\mathrm{BaNi}_{2}\left(\mathrm{PO}_{4}\right)_{2}$, where a dramatic temperaturedependent broadening was observed.

Finally, we note that studying the distributions of $t$ and $J$ in the form of the histograms in Fig. 7, and characterizing these by their mean and width, neglects bond correlations arising from the motion of the $\mathrm{Cu}$ ions. It is clear that one $\mathrm{Cu}$ atom moving towards $+x$ increases the value of $J_{\langle i, i+x\rangle}$ while simultaneously reducing $J_{\langle i-x, i\rangle}$, and similar correlations can be expected between the $x$ and $y$ directions. Because we use a finite lattice of frozen $J$ values, our calculations of the dynamical structure factor include these bond correlations. Their effects can be gauged by comparing the results in Fig. 9(a) with those obtained from a randomized set of lattice bond strengths. We find that this procedure gives barely discernible increases in the linewidths (data not shown) and, hence, comparisons with a purely Gaussian broadening are fully justified. We expect that this very small narrowing represents the full extent of correlation effects at the temperatures of interest here, where the Born approximation remains intact and ionic motion is essentially incoherent. However, at higher lattice energies, a deeper analysis of their consequences for electronic and magnetic properties would include phonon-mediated hopping and the possible enhancement of polaronic physics, which are topics lying beyond the scope of the current paper.

We conclude our detailed discussion with a more general comment. Zero-point and thermal ionic motion are present in all materials, and hence similar fluctuations can be expected in electronic hopping amplitudes and magnetic exchange interactions in a large class of quantum magnets, superconductors, multiferroics, and other strongly correlated materials. There are two reasons why the effects of positional fluctuations in $\mathrm{La}_{2} \mathrm{CuO}_{4}$, as studied in this paper, may be easier to quantify than a more general system, namely, the rather simple $\mathrm{Cu}-\mathrm{O}-\mathrm{Cu}$ bonding geometry, which has already been studied in great detail for planar cuprates, and the large energy scale for electronic and magnetic processes, which justifies the adiabatic approximation. However, there is no general argument in the theoretical analysis for ionic motion effects to be anomalously large in cuprates, and thus we would expect to see similar effects, namely, at the 10-20\% level in linewidths, in spectroscopic studies of $S(\mathbf{q}, \omega)$ and $A(\mathbf{k}, \omega)$ in many correlated magnetic and electronic systems. An accurate treatment of materials with more complex crystal structures may require $a b$ initio or quantum chemistry techniques to address the exchange paths (or hopping integrals) and their dependence on ionic position, and the possible breakdown of the adiabatic assumption may require treating the ionic motion in terms of coherent phonon modes.

\section{SUMMARY}

We have studied the quantum and thermal contributions to ionic motion in the antiferromagnetic insulator $\mathrm{La}_{2} \mathrm{CuO}_{4}$ by means of high-resolution neutron-diffraction experiments. We found that the anisotropic deformation of the ellipsoids of ionic motion is different for in-plane and out-of-plane ions. The elongation is most pronounced along the $z$ direction for $\mathrm{Cu}$ and $\mathrm{O} 1$ ions, while in-plane $\mathrm{O}$ motion reflects its bond orientation; by contrast, for the La and $\mathrm{O} 2$ ions, one sees at $290 \mathrm{~K}$ that in-plane motion is stronger. Zero-point, or quantum, fluctuations account for more than half of the ionic motion observed at $290 \mathrm{~K}$.

By using samples of $100 \%{ }^{16} \mathrm{O}$ and $78 \%{ }^{18} \mathrm{O}$ substitution, we investigated the influence of the oxygen isotope on the structural and thermal parameters of $\mathrm{La}_{2} \mathrm{CuO}_{4}$. We found a nonvanishing negative OIE on the lattice parameters of approximately $0.01 \%$. The negative OIE on $c$ is in qualitative agreement with the positive OIE we measured in the Néel temperature $T_{N}$. The OIE on the $\mathrm{Cu}-\mathrm{O} 1$ bond length is also negative. Further, our results demonstrate a considerable OIE on the zero-point motion, which is positive for the $\mathrm{La}$ and $\mathrm{Cu}$ atoms but negative for the $\mathrm{O} 1$ and $\mathrm{O} 2$ atoms. The preferred directions of ionic motion are identical for the ${ }^{16} \mathrm{O}$ and ${ }^{18} \mathrm{O}$ samples.

Working within the Born approximation, we use our detailed structural data to perform a theoretical study of how the zero-point and thermal motion of $\mathrm{Cu}$ and $\mathrm{O}$ ions will affect the electronic and magnetic properties of cuprates through the effective hopping integral $t$ and the antiferromagnetic exchange parameter $J$. By modeling the spatial distribution of ionic positions, we demonstrate that $J$ undergoes a Gaussian broadening, which is significant (exceeding 10\%) even at low temperatures and can be of the order of $20 \%$ at room temperature. This broadening shows a measurable positive $\mathrm{OIE}$ as a consequence of heavier $\mathrm{O}$ ions allowing enhanced $\mathrm{Cu}$ ion motion.

Our results suggest that this broadening cannot be neglected in the detailed theoretical modeling of cuprate systems. To illustrate this, we compute one static quantity and one dynamical one. The staggered magnetization is reduced only weakly by motional renormalization, changing less than the shift in the average of the $J$ distribution and thereby showing only percent-level effects. However, the transverse dynamical structure factor shows line broadening and peak intensity loss at the $10 \%$ level due to zero-point ionic motion, on top of which thermal effects cause strong additional softening and broadening; at $290 \mathrm{~K}$, the combination of thermal motional and spin fluctuations can lead to changes by a factor of two. These important renormalization effects, obtained within the approximation of incoherent ionic motion, indicate that models based on a static lattice may be insufficient for a full description of the electronic and magnetic properties of cuprates and, by extension, of other transition-metal compounds.

\section{ACKNOWLEDGMENTS}

Neutron powder-diffraction results are based on experiments performed at the Swiss spallation neutron source SINQ at the Paul Scherrer Institute, Villigen, Switzerland. We thank H. Keller and B. Batlogg for fruitful discussions, B. Rössner for helpful contributions, and S. M. Hayden for providing information on the resolution of previous neutron-scattering experiments. We are especially grateful to A. Chernyshev for detailed comments on the paper. This work was supported by the NCCR MaNEP and the Synergia network on Mott Physics Beyond the Heisenberg Model and Fellowship No. P2EZP2_148737 of the 
Swiss NSF; by the Danish Agency for Science, Technology, and Innovation under DANSCATT; by the NSF of China under Grant No. 11174365 and the National Basic Research Program of the Chinese MoST under Grant No. 2012CB921704; and by the Norwegian Research Council under NOTUR Grant No. nn4563k for using the Abel computer cluster.
[1] N. M. Plakida, High-Temperature Cuprate Superconductors: Experiment, Theory, and Applications (Springer, Heidelberg, 2010).

[2] M. A. Kastner, R. J. Birgeneau, G. Shirane, and Y. Endoh, Rev. Mod. Phys. 70, 897 (1998).

[3] D. A. Huse, Phys. Rev. B 37, 2380 (1988).

[4] R. Coldea, S. M. Hayden, G. Aeppli, T. G. Perring, C. D. Frost, T. E. Mason, S.-W. Cheong, and Z. Fisk, Phys. Rev. Lett. 86, 5377 (2001).

[5] H. M. Rønnow, D. F. McMorrow, R. Coldea, A. Harrison, I. D. Youngson, T. G. Perring, G. Aeppli, O. Syljuåsen, K. Lefmann, and C. Rischel, Phys. Rev. Lett. 87, 037202 (2001).

[6] P. W. Anderson, Science 235, 1196 (1987).

[7] J. Kohanoff, W. Andreoni, and M. Parrinello, Phys. Rev. B 46, 4371 (1992).

[8] E. Maxwell, Phys. Rev. 78, 477 (1950).

[9] C. A. Reynolds, B. Serin, W. H. Wright, and L. B. Nesbitt, Phys. Rev. 78, 487 (1950).

[10] H. Fröhlich, Proc. Phys. Soc. A 63, 778 (1950).

[11] P. S. Häfliger, A. Podlesnyak, K. Conder, E. Pomjakushina, and A. Furrer, Phys. Rev. B 74, 184520 (2006),

[12] H. Keller in Superconductivity in Complex Systems: Structure and Bonding, edited by K. A. Müller and A. Bussmann-Holder (Springer, Berlin, 2005), Vol. 114, p. 143.

[13] R. Khasanov, A. Shengelaya, D. Di Castro, E. Morenzoni, A. Maisuradze, I. M. Savic, K. Conder, E. Pomjakushina, A. Bussmann-Holder, and H. Keller, Phys. Rev. Lett. 101, 077001 (2008).

[14] A. Shengelaya, G. M. Zhao, C. M. Aegerter, K. Conder, I. M. Savic, and H. Keller, Phys. Rev. Lett. 83, 5142 (1999).

[15] A. Bussmann-Holder and H. Keller, in Polarons in Advanced Materials, edited by A. S. Alexandrov (Springer, Dordrecht \& Canopus, Bristol, 2007), p. 599.

[16] G. M. Zhao, K. K. Singh, and D. E. Morris, Phys. Rev. B 50, 4112 (1994).

[17] K. Hanzawa, J. Soc. Phys. Jpn 64, 4856 (1995).

[18] K. Conder, Mater. Sci. Eng. R 32, 41 (2001).

[19] K. Conder, G. M. Zhao, and R. Khasanov, Phys. Rev. B 66, 212409 (2002).

[20] P. Fischer, Physica B 276-278, 146 (2000).

[21] W. E. Fischer, Physica B 234-236, 1202 (1997).

[22] P. G. Radaelli, D. G. Hinks, A. W. Mitchell, B. A. Hunter, J. L. Wagner, B. Dabrowski, K. G. Vandervoort, H. K. Viswanathan, and J. D. Jorgensen, Phys. Rev. B 49, 4163 (1994).

[23] J. Rodríguez-Carvajal, Physica B 192, 55 (1993).

[24] We thank A. L. Chernyshev for pointing this out to us.

[25] J. M. Ziman, Principles of the Theory of Solids, 2nd ed. (Cambridge University Press, Cambridge, 1972), Chap. 2.

[26] J. D. Jorgensen, B. Dabrowski, S. Pei, D. G. Hinks, L. Soderholm, B. Morosin, J. E. Schirber, E. L. Venturini, and D. S. Ginley, Phys. Rev. B 38, 11337 (1988).

[27] C. Chaillout, J. Chenavas, S. W. Cheong, Z. Fisk, M. Marezio, B. Morosin, and J. E. Schirber, Physica C 170, 87 (1990).
[28] H. B. Rosenstock, Phys. Rev. 129, 1959 (1963).

[29] F.-C. Zhang and T. M. Rice, Phys. Rev. B 37, 3759 (1988).

[30] H. Eskes and J. H. Jefferson, Phys. Rev. B 48, 9788 (1993).

[31] P. Böni, J. D. Axe, G. Shirane, R. J. Birgeneau, D. R. Gabbe, H. P. Jenssen, M. A. Kastner, C. J. Peters, P. J. Picone, and T. R. Thurston, Phys. Rev. B 38, 185 (1988).

[32] K.-P. Bohnen, R. Heid, and M. Krauss, Europhys. Lett. 64, 104 (2003).

[33] A. Lanzara, P. V. Bogdanov, X. J. Zhou, S. A. Kellar, D. L. Feng, E. D. Lu, T. Yoshida, H. Eisaki, A. Fujimori, K. Kishio, J. I. Shimoyama, T. Noda, S. Uchida, Z. Hussain, and Z. X. Shen, Nature (London) 412, 510 (2001).

[34] S. Bramwell, J. Phys. Condens. Matter 2, 7527 (1990).

[35] W. A. Harrison, Orbital Overlap and the Chemical Bond (Wiley, New York, 1980).

[36] J. H. Jefferson, H. Eskes, and L. F. Feiner, Phys. Rev. B 45, 7959 (1992).

[37] V. I. Belinicher and A. L. Chernyshev, Phys. Rev. B 49, 9746 (1994).

[38] M. C. Aronson, S. B. Dierker, B. S. Dennis, S.-W. Cheong, and Z. Fisk, Phys. Rev. B 44, 4657 (1991).

[39] H. M. Rønnow, D. F. McMorrow, and A. Harrison, Phys. Rev. Lett. 82, 3152 (1999).

[40] G. M. Zhao, H. Keller, and K. Conder, J. Phys. Condens. Matt. 13, R569 (2001), and references therein.

[41] C. Bernhard, T. Holden, A. V. Boris, N. N. Kovaleva, A. V. Pimenov, J. Humlicek, C. Ulrich, C. T. Lin, and J. L. Tallon, Phys. Rev. B 69, 052502 (2004).

[42] G.-H. Gweon, T. Sasagawa, S. Y. Zhou, J. Graf, H. Takagi, D.-H. Lee, and A. Lanzara, Nature (London) 430, 187 (2004).

[43] N. B. Christensen, D. F. McMorrow, H. M. Rønnow, A. Harrison, T. G. Perring, and R. Coldea, J. Magn. Magn. Mater. 272-274, 896 (2004).

[44] N. B. Christensen, H. M. Rønnow, D. F. McMorrow, A. Harrison, T. G. Perring, M. Enderle, R. Coldea, L. P. Regnault, and G. Aeppli, Proc. Natl. Acad. Sci. 104, 15264 (2007).

[45] N. Tsyrulin, F. Xiao, A. Schneidewind, P. Link, H. M. Rønnow, J. Gavilano, C. P. Landee, M. M. Turnbull, and M. Kenzelmann, Phys. Rev. B 81, 134409 (2010).

[46] O. F. Syljuåsen and A. W. Sandvik, Phys. Rev. E. 66, 046701 (2002).

[47] A. W. Sandvik, Phys. Rev. B 57, 10287 (1998).

[48] O. F. Syljuåsen, Phys. Rev. B 78, 174429 (2008).

[49] O. F. Syljuåsen and H. M. Rønnow, J. Phys.: Condens. Matter 12, L405 (2000).

[50] H. M. Rønnow, D. F. McMorrow, A. Harrison, I. D. Youngson, R. Coldea, T. G. Perring, G. Aeppli, and O. Syljuåsen, J. Magn. Magn. Mater. 236, 4 (2001).

[51] N. S. Headings, S. M. Hayden, R. Coldea, and T. G. Perring, Phys. Rev. Lett. 105, 247001 (2010).

[52] M. Guarise, B. Dalla Piazza, M. Moretti Sala, G. Ghiringhelli, L. Braicovich, H. Berger, J. N. Hancock, D. van der Marel, 
T. Schmitt, V. N. Strocov, L. J. P. Ament, J. van den Brink, P.-H. Lin, P. Xu, H. M. Rønnow, and M. Grioni, Phys. Rev. Lett. 105, 157006 (2010).

[53] B. Dalla Piazza, M. Mourigal, M. Guarise, H. Berger, T. Schmitt, K. J. Zhou, M. Grioni, and H. M. Rønnow, Phys. Rev. B 85, 100508 (2012).

[54] T. P. Devereaux and R. Hackl, Rev. Mod. Phys. 79, 175 (2007).
[55] K. B. Lyons, P. E. Sulewski, P. A. Fleury, H. L. Carter, A. S. Cooper, G. P. Espinosa, Z. Fisk, and S.-W. Cheong, Phys. Rev. B 39, 9693 (1989).

[56] C. M. Canali and S. M. Girvin, Phys. Rev. B 45, 7127 (1992).

[57] A. A. Katanin and A. P. Kampf, Phys. Rev. B 67, 100404 (2003).

[58] A. L. Chernyshev, M. E. Zhitomirsky, N. Martin, and L.-P. Regnault, Phys. Rev. Lett. 109, 097201 (2012). 